\title{
Rare Germline Genetic Variants and the Risks of Epithelial Ovarian Cancer
}

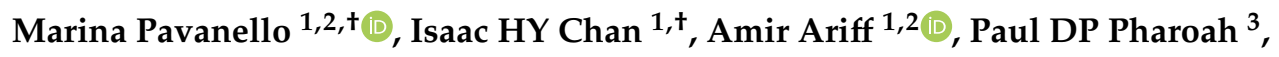 \\ Simon A. Gayther ${ }^{4,5}$ and Susan J. Ramus ${ }^{1,2, *}$ \\ 1 School of Women's and Children's Health, Faculty of Medicine, University of New South Wales, \\ Sydney 2052, Australia; m.pavanello@student.unsw.edu.au (M.P.); isaac.chan@unsw.edu.au (I.H.C.); \\ amir.ariff@unsw.edu.au (A.A.) \\ 2 Adult Cancer Program, Lowy Cancer Research Centre, University of New South Wales, \\ Sydney 2052, Australia \\ 3 Strangeways Research Laboratory, University of Cambridge, Cambridge CB1 8RN, UK; \\ pp10001@medschl.cam.ac.uk \\ 4 Center for Cancer Prevention and Translational Genomics, Cedars Sinai Medical Center, \\ Los Angeles, CA 90048, USA; simon.gayther@cshs.org \\ 5 Applied Genomics, Computation and Translational Core, Cedars Sinai Medical Center, \\ Los Angeles, CA 90048, USA \\ * Correspondence: s.ramus@unsw.edu.au \\ + These authors have contributed equally to this work.
}

Received: 16 September 2020; Accepted: 14 October 2020; Published: 19 October 2020

check for updates

Simple Summary: Several genes have been confirmed as risk genes for epithelial ovarian cancer (EOC). There are five main types of EOC, with different molecular changes and clinical characteristics, suggesting they should be considered different diseases. This review summarises the contribution of rare inherited mutations to EOC susceptibility, focussing on the frequency in each EOC type. Susceptibility genes can have a major clinical impact, reducing ovarian cancer incidence by screening of family members to detect women at higher risk than the general population. They can also lead to the development of new targeted treatments.

Abstract: A family history of ovarian or breast cancer is the strongest risk factor for epithelial ovarian cancer (EOC). Germline deleterious variants in the BRCA1 and BRCA2 genes confer EOC risks by age 80 , of $44 \%$ and $17 \%$ respectively. The mismatch repair genes, particularly $M S H 2$ and $M S H 6$, are also EOC susceptibility genes. Several other DNA repair genes, BRIP1, RAD51C, RAD51D, and PALB2, have been identified as moderate risk EOC genes. EOC has five main histotypes; high-grade serous (HGS), low-grade serous (LGS), clear cell (CCC), endometrioid (END), and mucinous (MUC). This review examines the current understanding of the contribution of rare genetic variants to EOC, focussing on providing frequency data for each histotype. We provide an overview of frequency and risk for pathogenic variants in the known susceptibility genes as well as other proposed genes. We also describe the progress to-date to understand the role of missense variants and the different breast and ovarian cancer risks for each gene. Identification of susceptibility genes have clinical impact by reducing disease-associated mortality through improving risk prediction, with the possibility of prevention strategies, and developing new targeted treatments and these clinical implications are also discussed.

Keywords: ovarian cancer risk; rare germline variants; susceptibility genes 


\section{Introduction}

Epithelial ovarian cancer (EOC) is the seventh most common cancer in women worldwide, with over 295,000 incident cases each year, and is the leading cause of mortality relating to gynaecological malignancies, with 184,000 deaths each year [1]. Typically, EOCs are stratified into five main histological subtypes: High grade serous, which account for up to $70 \%$ of all EOC cases, endometrioid $(\sim 10 \%)$, clear cell $(\sim 10 \%)$, mucinous $(\sim 3 \%)$, and low-grade serous carcinomas $(<5 \%)$ [2]. The five different histotypes have different risk factors and molecular characteristics. The inherited genetics of each histotype is also likely to be different.

The prognosis of ovarian cancer is poor, with a five-year survival rate of just $47 \%$ [3]. Ovarian cancer is difficult to diagnose early in its disease course, and $80 \%$ of cases are diagnosed after extensive metastasis at stage III or IV, which carry a five-year survival rate of $41 \%$ and $20 \%$ respectively [3]. However, outcomes are good if ovarian cancer is detected early, with an $89 \%$ five-year survival in stage I cancers [3]. Screening for ovarian cancer is of limited utility and no studies investigating ovarian cancer screening have shown a significant impact of screening on mortality [4,5]. As a result, population-based screening for ovarian cancer is not recommended in the guidelines of any major society. As the prognosis of EOC is related to its stage at diagnosis, the ability to use genetic information to predict EOC risk and intervene before disease development would reduce overall mortality and morbidity in ovarian cancer [6].

A family history of EOC confers an increase in relative risk (RR) of ovarian cancer of 2.96. The known EOC risk genes explain less than half of the excess familial risk for EOC, suggesting that there are still undiscovered ovarian cancer predisposing genes to be found $[7,8]$. The known ovarian cancer predisposing genes are from two different DNA repair pathways. The homologous recombination (HR) DNA repair pathway and the mismatch repair (MMR) DNA pathway. The majority of the genes are part of the HR DNA repair pathway. BRCA1 and $B R C A 2$ have been confirmed as highly penetrant predisposition genes for EOC [9]. Several other genes-BRIP1, RAD51C, RAD51D, and PALB2, recently described as moderate risk genes, are also part of this pathway [10-12]. The mismatch repair genes MLH1, MSH2, MSH6, and PSM2 are also confirmed moderate risk EOC susceptibility genes [13]. The frequency of disease-associated variants in these genes is different for the different EOC histotypes. Thirty-seven common variants have been identified for ovarian cancer, using genome-wide association studies (GWAS) $[14,15]$. The ability to derive accurate risk estimates for ovarian cancer from genetic information in asymptomatic women has significant implications for ovarian cancer management.

This review aims to summarise the progress to date in efforts to understand the contribution of rare genetic variants to ovarian cancer and histotype specific differences, as well as the clinical implications of these discoveries. In the first part of this review, we describe germline protein truncating variants (insertion/deletions or nonsense) in confirmed EOC susceptibility genes, followed by an overview of the role of missense variants in these genes. Lastly, we described germline protein truncating variants in other proposed genes that have not been validated as EOC susceptibility genes.

\section{Protein Truncating Variants in Confirmed Susceptibility Genes}

\subsection{BRCA1 and $B R C A 2$}

These two major breast/ovarian cancer susceptibility genes encode proteins that work in DNA replication pathways to avoid double-strand DNA damage. Deleterious protein truncating variants include small insertions and deletions and single base changes, as well as large genomic alterations, which account for approximately $10 \%$ of $B R C A 1$ variants [16]. Several deleterious missense variants have also been described.

The prevalence and penetrance of deleterious variants in $B R C A 1$ and $B R C A 2$ has been extensively studied in ovarian cancer patients. The case ascertainment, either from families with a history of breast or ovarian cancer or from the general ovarian cancer population, has a major impact on the results. The presence of founder mutations in the population also affects the prevalence and has important 
implications for clinical testing. The presence of founder mutations can allow for cheap and rapid testing and the potential for population screening. In the Ashkenazi Jewish population, there are three $B R C A$ founder mutations (185delAG and 5382insC in BRCA1 and 6174delT in BRCA2) that accounts for almost all mutations present in this population [17]. In the Icelandic population, there is a single founder mutation, $999 \mathrm{del} 5$, in the $B R C A 2$ gene. Most of the breast and ovarian cancer cases in Icelandic families have this $B R C A 2$ founder mutation, while mutations in $B R C A 1$ are very rare [18]. Founder mutations in the $B R C A$ genes have also been reported in many other populations, including Russians [19,20], Polish [21], Norwegian [22], Finnish [23], Japanese [24], and Chinese [25]. While some mutations are restricted to isolated regions or certain populations, others are common across different countries, such as BRCA1 5382insC mutation that is common in many European populations [17].

Early studies of cases selected for a family history of breast or ovarian cancer found that $24-76 \%$ had deleterious variants in BRCA1 and 1-17\% had deleterious variants in BRCA2 [17]. Recent studies using clinical testing laboratory data have reported the prevalence of $B R C A 1 / 2$ pathogenic variants in 5020 and 7489 cases from the USA [26,27], 4409 cases from France [28], and 3230 cases from a meta-analysis of 48 multi-gene panel testing-based studies [29]. In these studies, the frequency of germline pathogenic variants in $B R C A 1$ in ovarian cancer cases were $5.1 \%, 3.6 \%, 3.7 \%$, and $8.6 \%$, respectively, and 3.9\%, 3.3\%, 4.0\%, and 4.5\% in BRCA2 [26-29].

Testing of unselected ovarian cancer cases in non-Ashkenazi Jewish populations shows that the frequency of deleterious $B R C A 1$ and $B R C A 2$ variants ranged from $3-10 \%$ and $0.6-6 \%$, respectively [17]. Recent population-based studies from Table 1 found that $3.8-11.1 \%$ of cases have $B R C A 1$ deleterious variants and $4.3-6.4 \%$ had $B R C A 2$ deleterious variants.

In a large prospective study of $B R C A$ carriers ascertained through family clinics, the cumulative risk, by age 80 , of ovarian cancer in women with pathogenic variants in BRCA1 and BRCA2 was estimated to be $44 \%$ (95\% confidence interval (CI), 36-53\%) and $17 \%(95 \%$ CI, $11-25 \%)$, respectively [9]. The histotypes of ovarian cancer diagnosed in the study were not described [9]. Breast cancer was also assessed and the cumulative risk at the age of 80 years was estimated to be $72 \%$ (95\% CI, $65-79 \%)$ for $B R C A 1$ carriers and $69 \%(95 \% \mathrm{CI}, 61-77 \%)$ for $B R C A 2$ [9]. Variants in these $B R C A$ genes are also associated with increased risk of pancreatic cancer [30] and high-grade prostate cancer [31].

Four studies, with cases unselected for age or family history, provided information on histotype (Table 1). Due to the small numbers of the much rarer histotypes (END, CCC, LGS, and MUC), the frequencies of germline pathogenic variants varied significantly. In an effort to estimate the frequency in these rarer histotypes, we have combined the results across studies. This showed that the frequency of deleterious variants in BRCA1 were approximately $7.8 \%$ in HGS, $3 \%$ in END, $3.6 \%$ in CCC, $3.7 \%$ in LGS, and $<1 \%$ in MUC [32-35]. In BRCA2, deleterious variants were seen in approximately $5.9 \%$ of HGS cases, $2.9 \%$ of END, $0.9 \%$ of CCC, $2.0 \%$ of LGS, and $<1 \%$ of MUC [33-35] (Table 1). According to these data, both genes have a higher frequency of variants in the HGS histotype compared to the non-HGS histotypes, and a much lower frequency in mucinous cases. Available cumulative risks from these retrospective studies are comparable to the prospective study previously mentioned [9] (Table 1).

Ovarian cancer patients with deleterious variants in $B R C A 1$ and $B R C A 2$ are characterized by genomic instability within their tumours. They have a better response to platinum-based chemotherapies, resulting in improved five-year overall survival. Five-year overall survival for non-carriers was reported to be $36 \%$ (95\% CI 34-38) compared to $44 \%$ (95\% CI 40-48) for BRCA1-carriers and $52 \%(95 \%$ CI $46-58)$ for $B R C A 2$-carriers [36]. However, this survival advantage was not present when follow-up was extended to 10 years $[37,38]$.

Identifying pathogenic variants associated with increased risk of ovarian cancer has major clinical implications. Due to the magnitude of ovarian cancer risk associated with variants in BRCA1 and $B R C A 2$, risk-reducing salpingo-oophorectomy (RRSO) is currently recommended as a prevention strategy for $B R C A 1$ carriers by age 35 to 40 years, once the woman's childbearing is complete, and for $B R C A 2$ carriers by age 40 to 45 [39]. 
Table 1. Frequency of germline pathogenic variants in BRCA1/2 in epithelial ovarian cancer (EOC) histotypes, in cases from population studies.

\begin{tabular}{|c|c|c|c|c|c|c|c|c|c|c|c|c|}
\hline \multirow{2}{*}{ Gene } & \multirow{2}{*}{ Study } & \multicolumn{7}{|c|}{$\begin{array}{c}\text { Cases } \\
\text { Carriers/Total (Frequency) }\end{array}$} & \multicolumn{2}{|c|}{$\begin{array}{c}\text { Controls } \\
\text { Carriers/Total (Frequency) }\end{array}$} & \multirow{2}{*}{$\begin{array}{c}\text { OR } \\
(95 \% \mathrm{CI})\end{array}$} & \multirow{2}{*}{$\begin{array}{c}\text { Cumulative Risk } \\
\qquad(95 \% \mathrm{CI})^{\mathrm{a}}\end{array}$} \\
\hline & & HGS & END & $\mathrm{CCC}$ & LGS & MUC & $\begin{array}{l}\text { Mixed/ } \\
\text { Unk }^{\text {b }}\end{array}$ & All EOC & Matched & Publicly Available & & \\
\hline \multirow{5}{*}{$B R C A 1$} & $\begin{array}{l}\text { Walsh et al., } \\
2011[34]\end{array}$ & $\begin{array}{c}31 / 242 * * \\
(12.8 \%)\end{array}$ & $\begin{array}{c}2 / 23 \\
(8.7 \%)\end{array}$ & $\begin{array}{c}1 / 17 \\
(5.9 \%)\end{array}$ & $* *$ & - & $\begin{array}{l}6 / 78 \\
(7.7 \%)\end{array}$ & $\begin{array}{c}40 / 360 \\
(11.1 \%)\end{array}$ & - & - & NC & $\mathrm{NC}$ \\
\hline & $\begin{array}{l}\text { Alsop et al., } \\
2012^{+}[32]\end{array}$ & $\begin{array}{c}74 / 709^{* *} \\
(10.4 \%)\end{array}$ & $\begin{array}{l}7 / 119 \\
(5.9 \%)\end{array}$ & $\begin{array}{c}4 / 63 \\
(6.3 \%)\end{array}$ & $* *$ & - & $\begin{array}{l}3 / 110 \\
(2.7 \%)\end{array}$ & $\begin{array}{l}88 / 1001 \\
(8.79 \%)\end{array}$ & - & - & NC & NC \\
\hline & $\begin{array}{l}\text { Song et al., } \\
2014^{\wedge}[33]\end{array}$ & $\begin{array}{c}58 / 1105^{*} \\
(5.2 \%)\end{array}$ & $\begin{array}{l}3 / 322 \\
(0.9 \%) \\
\end{array}$ & $\begin{array}{c}3 / 192 \\
(1.6 \%) \\
\end{array}$ & $\begin{array}{c}6 / 172 \\
(3.5 \%) \\
\end{array}$ & $\begin{array}{l}1 / 157 \\
(0.6 \%) \\
\end{array}$ & $\begin{array}{l}13 / 274 \\
(4.7 \%) \\
\end{array}$ & $\begin{array}{l}84 / 2222 \\
(3.78 \%) \\
\end{array}$ & $\begin{array}{l}1 / 1528 \\
(0.07 \%)\end{array}$ & - & $\begin{array}{c}60^{\#} \\
(10-2100) \\
\end{array}$ & $\begin{array}{c}61 \% \\
(15-99 \%) \\
\end{array}$ \\
\hline & $\begin{array}{l}\text { Norquist et al., } \\
2016[35]\end{array}$ & $\begin{array}{c}155 / 1498 \\
(10.3 \%)\end{array}$ & $\begin{array}{c}4 / 77 \\
(5.2 \%)\end{array}$ & $\begin{array}{c}4 / 58 \\
(6.9 \%) \\
\end{array}$ & $\begin{array}{c}3 / 70 \\
(4.3 \%)\end{array}$ & $\begin{array}{l}0 / 16 \\
(0 \%)\end{array}$ & $\begin{array}{l}16 / 196 \\
(8.2 \%)\end{array}$ & $\begin{array}{c}142 / 1915 \\
(7.41 \%)\end{array}$ & - & $\begin{array}{c}114 / 36276 \\
(0.31 \%)\end{array}$ & $\begin{array}{c}48.9 \\
(24-100)\end{array}$ & NC \\
\hline & Total & $\begin{array}{c}278 / 3554 \\
(7.82 \%)\end{array}$ & $\begin{array}{c}16 / 541 \\
(2.95 \%)\end{array}$ & $\begin{array}{l}12 / 330 \\
(3.63 \%)\end{array}$ & $\begin{array}{c}9 / 242 \\
(3.72 \%)\end{array}$ & $\begin{array}{c}1 / 173 \\
(0.58 \%)\end{array}$ & $\begin{array}{l}38 / 658 \\
(5.77 \%)\end{array}$ & $\begin{array}{c}354 / 5498 \\
(6.43 \%)\end{array}$ & $\begin{array}{c}1 / 1528 \\
(0.07 \%)\end{array}$ & - & - & - \\
\hline \multirow{5}{*}{$B R C A 2$} & $\begin{array}{l}\text { Walsh et al., } \\
2011[34]\end{array}$ & $\begin{array}{c}18 / 242^{* *} \\
(7.4 \%)\end{array}$ & $\begin{array}{l}0 / 23 \\
(0 \%)\end{array}$ & $\begin{array}{l}0 / 17 \\
(0 \%)\end{array}$ & $* *$ & - & $\begin{array}{c}5 / 78 \\
(6.4 \%)\end{array}$ & $\begin{array}{l}23 / 360 \\
(6.38 \%) \\
\end{array}$ & - & - & NC & NC \\
\hline & $\begin{array}{l}\text { Alsop et al., } \\
2012^{+}[32]\end{array}$ & $\begin{array}{c}44 / 709^{* *} \\
(6.2 \%)\end{array}$ & $\begin{array}{l}3 / 119 \\
(2.5 \%)\end{array}$ & $\begin{array}{l}0 / 63 \\
(0 \%)\end{array}$ & $* *$ & - & $\begin{array}{l}6 / 110 \\
(5.4 \%)\end{array}$ & $\begin{array}{l}53 / 1001 \\
(5.29 \%) \\
\end{array}$ & - & - & NC & NC \\
\hline & $\begin{array}{l}\text { Song et al., } \\
2014^{\wedge}[33]\end{array}$ & $\begin{array}{c}64 / 1105 * \\
(5.8 \%)\end{array}$ & $\begin{array}{l}10 / 322 \\
(3.1 \%)\end{array}$ & $\begin{array}{l}3 / 192 \\
(1.6 \%)\end{array}$ & $\begin{array}{l}4 / 172 \\
(2.3 \%)\end{array}$ & $\begin{array}{l}1 / 157 \\
(0.6 \%)\end{array}$ & $\begin{array}{l}12 / 274 \\
(4.4 \%)\end{array}$ & $\begin{array}{l}94 / 2222 \\
(4.23 \%) \\
\end{array}$ & $\begin{array}{l}4 / 1528 \\
(0.26 \%)\end{array}$ & - & $\begin{array}{c}177^{\#} \\
(6.3-63)\end{array}$ & $\begin{array}{c}24 \% \\
(10-62 \%) \\
\end{array}$ \\
\hline & $\begin{array}{l}\text { Norquist et al., } \\
2016 \text { [35] }\end{array}$ & $\begin{array}{c}85 / 1498 \\
(5.7 \%)\end{array}$ & $\begin{array}{c}3 / 77 \\
(3.9 \%)\end{array}$ & $\begin{array}{l}0 / 58 \\
(0 \%)\end{array}$ & $\begin{array}{c}1 / 70 \\
(1.4 \%)\end{array}$ & $\begin{array}{l}0 / 16 \\
(0 \%)\end{array}$ & $\begin{array}{c}9 / 196 \\
(4.6 \%)\end{array}$ & $\begin{array}{l}98 / 1915 \\
(5.11 \%) \\
\end{array}$ & - & $\begin{array}{c}149 / 36276 \\
(0.41 \%)\end{array}$ & $\begin{array}{c}14 \\
(8.2-23.8)\end{array}$ & NC \\
\hline & Total & $\begin{array}{c}211 / 3554 \\
(5.93 \%)\end{array}$ & $\begin{array}{l}16 / 541 \\
(2.95 \%)\end{array}$ & $\begin{array}{c}3 / 330 \\
(0.91 \%)\end{array}$ & $\begin{array}{l}5 / 242 \\
(2.0 \%)\end{array}$ & $\begin{array}{c}1 / 173 \\
(0.57 \%)\end{array}$ & $\begin{array}{l}32 / 658 \\
(4.86 \%)\end{array}$ & $\begin{array}{c}268 / 5498 \\
(4.87 \%)\end{array}$ & $\begin{array}{l}4 / 1528 \\
(0.26 \%)\end{array}$ & - & - & - \\
\hline
\end{tabular}

-: Not available, NC: Not calculated due to no controls or not described, ${ }^{* *}$ Frequency of serous combined, ${ }^{*}$ Frequency of HGS and serous undifferentiated, ${ }^{*}$ Odds ratio (OR) for large genomic alterations. Bold: highlight info. 
Furthermore, understanding the role of $B R C A 1 / 2$ variants in ovarian cancer has allowed for the development of targeted therapies, namely PARP (poly[adenosine diphosphate-ribose] polymerase) inhibitors, which improve progression-free survival in selected women with ovarian cancer. Approximately $50 \%$ of HGS cases present defects in DNA repair mechanisms due to pathogenic variants in the homologous recombination deficiency (HRD) genes, such as BRCA1/2, or due to functional inactivation through methylation [40]. PARPi have shown highly efficacious activity particularly in women with platinum-sensitive disease carrying BRCA1/2 variants, or women with other homologous recombination deficiencies [41-43]. Improved activity has also been seen in women with recurrent disease regardless of their $B R C A$ status [44]. As BRCA1/2 status affects clinical management of affected women, current guidelines recommend $B R C A 1 / 2$ testing in all non-mucinous ovarian cancer cases [39].

\subsection{BRIP1}

The protein encoded by BRIP1 is part of the Fanconi anaemia group (FANCJ) and is involved in the repair of DNA double-strand breaks by homologous recombination. BRIP1 was described as a candidate risk gene by Walsh et al. that identified germline loss-of-function variants in $1 \%$ of ovarian cancer cases not selected for age or family history [34]. Pathogenic germline variants in BRIP1 are the most common mutation found in ovarian cancer after $B R C A 1 / 2$ with a frequency of approximately $1 \%$ of the EOC cases [11].

The contribution of germline protein truncating variants in BRIP1 have not yet been assessed in prospective studies. Ramus et al. described a relative risk associated with protein truncating variants in BRIP1 of 11.2 (95\% CI 3.2-34.1), with an estimated cumulative risk of 5.8\% (95\% CI 3.6-9.1\%) by the age of 80 years [11] (Table 3). The association between protein truncating variants in BRIP1 and risk of ovarian cancer has been confirmed in other analyses (associated risks ranged from 2.6 to $6.4[27-29,35,45]$ ) (Tables 2 and 3). The frequency of protein truncating variants in population-based studies, separated by ovarian cancer histotypes are given in Table 3, and the frequency in the other retrospective studies, mostly family studies or with women referred to clinical testing, are given in Table 2. Some of the studies in Table 2 have included predicted deleterious missense changes that cannot be separated from the totals, and these are indicated.

Three studies have reported the frequency of protein truncating variants in BRIP1 by ovarian cancer histotype. Combining these data show that approximately $1.2 \%$ of HGS and END cases and $0.8 \%$ of LGS cases had protein truncating variants in BRIP1 [11,34,35] (Table 3). No variants were observed in CCC and MUC, but the total number of cases examined were low. Additional studies are needed to further assess the contribution of germline protein truncating variants in BRIP1 in the ovarian cancer histotypes.

Available data for BRIP1 consistently have shown an increased risk of ovarian cancer, with cumulative risk estimated to be approximately $6 \%$ by age of 80 , and the National Comprehensive Cancer Network, USA, now recommends RRSO for women, starting from ages 45 to 50 [39].

\subsection{RAD51C and RAD51D}

RAD51C and RAD51D are homologous recombination genes, which encode proteins that interact with $B R C A 1 / 2$ and participate in the DNA repair process. Ovarian cancer risk attributed to protein truncating variants in $R A D 51 C$ and $R A D 51 D$ was first described by Meindl et al. in 2010 and Loveday et al. in 2011 [46,47].

Song et al. and Norquist et al. provided the frequency of protein truncating variants in the $R A D 51 C / D$ genes in population-based studies by ovarian cancer histotypes [35,48] (Table 3). Combining their data, approximately $0.4-0.5 \%$ of HGS, END and CCC cases and $0.2 \%$ of LGS had protein truncating variants in RAD51C. For RAD51D, $0.5 \%$ of HGS cases and $0.9 \%$ of END cases had protein truncating variants. These variants were not detected in CCC, LGS, and MUC cases, although so far, the number of cases of these histotypes in the datasets are low. The frequency of deleterious variants in family-based studies that combined all EOC cases are given in Table 2. The estimated 
risk associated with $R A D 51 C / D$ described in the two population-studies were OR 5.2 (95\% CI 1.1-24) and 3.4 (95\% CI 1.5-7.6) for RAD51C and 12.0 (95\% CI 1.5-90) and 10.9 (95\% CI 4.6-26) for RAD51D (Table 3) $[35,48]$. Three additional case-control studies which included only individuals referred to clinical testing estimated a risk of OR 4.9 (95\%CI 3.0-8.0), OR 14.6 (95\% CI 5.3 -29.5), and SRR 5.12 (95\% CI 3.7-6.9) for RAD51C. For RAD51D, associated risks were OR 4.78 (95\% CI 2.1-10.7), OR 11.8 (95\% CI 1.1-40), and SRR 6.34 (95\% CI 3.1-11.3) (Table 2) [26-28].

More recently, Yang et al. performed a study of 125 families with pathogenic variants in $R A D 51 C$ and 60 families with pathogenic variants in $R A D 51 D$ and confirmed an increased risk of ovarian cancer associated with pathogenic variants in both genes (RAD51C, RR 7.55, 95\% CI 5.6-10.2 and RAD51D, RR 7.6, 95\% CI 5.6-10.3) [10]. The cumulative risk of having ovarian cancer by age 80 was estimated to be $11 \%$ (95\% CI 6-21\%) for RAD51C and 13\% (95\% CI 7-23\%) for RAD51D based on their segregation analysis [10] (Table 2). RAD51C/D were also shown to be associated with breast cancer with cumulative risk of $21 \%$ (95\% CI 15-29\%) and 20\% (95\% CI 14-28\%) for these genes by the age of 80 years, respectively [10].

There is no consensus about the EOC risk threshold for surgical prevention, although the acceptability of RRSO for women with a lifetime risk greater than $10 \%$ is well-established. It has been recently suggested that this threshold should be lower and was demonstrated that prophylactic surgery is cost-effective for women at lifetime risk of 5\% [49-51]. Considering the current cumulative risk estimates for $R A D 51 C$ and $R A D 51 D$, women carrying pathogenic variants in these genes could be offered preventive surgery starting from ages 45 to 50 , once childbearing is complete.

\subsection{PALB2}

PALB2 is a confirmed breast cancer susceptibility gene with a cumulative risk estimated to be $44 \%$ by 80 years [52], and therefore clinical testing for germline pathogenic variants in this gene is part of breast cancer standard of care. PALB2 is needed to recruit BRCA2 in the HR DNA repair pathway [53].

Initial analysis of 3227 EOC cases and 3444 matched-controls suggested that larger numbers of samples were required to determine if PALB2 was an ovarian cancer susceptibility gene ( $\mathrm{p} 0.08)$ [11]. Evidence of risk association was observed in case-control analysis of 1915 EOC cases compared to publicly available controls with the OR estimated to be 4.4 (95\% CI 2.1-9.1) [35]. Recently, PALB2 has been confirmed as a risk gene by targeted sequencing in 5123 HGS cases and 5202 controls, WES data from 829 cases and 913 controls, and genotyping data from an independent set of $\sim 14,000$ EOC cases and 29,000 controls [54]. The odds ratio was estimated to be 3.01 (95\% CI 1.59-5.68) [54] (Table 3).

Histotype data are only available from the first two studies, and combined show that approximately $0.4 \%$ of HGS cases had protein truncating variants in PALB2 (Table 3). A lower frequency was found for LGS $(0.2 \%)$ and no variants were found in END and MUC. In contrast CCC cases had a frequency of $2.4 \%$. The total number of non-HGS cases examined to date, particularly for CCC, is very low, thus larger numbers are needed to establish the true frequency of PALB2 pathogenic variants in the rarer histotypes.

An increased risk of ovarian cancer has recently been confirmed in a study of 976 individuals with protein truncating variants in PALB2 from 524 families, where complex segregation analysis adjusted for ascertainment was performed. The relative risk was estimated to be 2.91 (95\% CI 1.4-6.0) [12] (Table 2). Cumulative risks were estimated to be approximately 5\% (95\% CI 2-10\%) by the age of 80 years in the family-based study [12] and 3.2\% (95\% CI 1.8-5.7\%) by the same age in the case-control study previously described [54]. Discussions in the clinical community on whether or not these women should be eligible for prophylactic surgery are still ongoing.

Yang et al. also showed that protein truncating variants in PALB2 were associated with increased risk of female and male breast cancer (RR 7.18 95\% CI 5.8-8.8 and 7.34 95\% CI 1.2-42.8, respectively) and pancreatic cancer (RR 2.37 95\% CI 1.2-4.5) [12]. 
Table 2. Frequency of germline pathogenic variants in BRIP1, RAD51C, RAD51D, and PALB2, in EOC cases from family history studies.

\begin{tabular}{|c|c|c|c|c|c|c|c|}
\hline \multirow{2}{*}{ Gene } & \multirow{2}{*}{ Study } & \multirow{2}{*}{ Type of Study } & \multirow{2}{*}{$\begin{array}{c}\begin{array}{c}\text { Cases Carriers/Total } \\
\text { (Frequency) }\end{array} \\
\text { All EOC }\end{array}$} & \multicolumn{2}{|c|}{$\begin{array}{c}\text { Controls } \\
\text { (Carriers/Total Frequency) }\end{array}$} & \multirow{2}{*}{ Risk $(95 \%$ CI) } & \multirow{2}{*}{$\begin{array}{c}\text { Cumulative Risk } \\
\qquad(95 \% \mathrm{CI})\end{array}$} \\
\hline & & & & Matched & $\begin{array}{l}\text { Publicly } \\
\text { Available }\end{array}$ & & \\
\hline \multirow{4}{*}{ BRIP1 } & Lilyquist et al., $2017^{\mathbb{I I}}$ [27] & Clinical testing lab & $58 / 6294(0.92 \%)$ & - & ExAC & SRR: 4.99 (3.8-6.4) & $\mathrm{NC}$ \\
\hline & Kurian et al., $2017^{\mathbb{I}}[26]$ & Clinical testing lab & $36 / 5020(0.71 \%)$ & $161 / 64,649(0.24 \%)$ & - & OR: $2.62(1.7-3.9)$ & $\mathrm{NC}$ \\
\hline & Castera et al., $2018 \mathbb{I}$ [28] & Genetic counselling & $21 / 4408(0.48 \%)$ & - & $72 / 36,276^{\text {a }}(0.20 \%)$ & OR: 3.77 (0.7-9.4) & $\mathrm{NC}$ \\
\hline & Suszynska et al., 2020 [45] & Meta-analysis^ & $200 / 22,494(0.89 \%)$ & - & $\begin{array}{c}209 / 115,375^{b} \\
(0.18 \%)\end{array}$ & OR: $4.94(4.0-6.0)$ & $\mathrm{NC}$ \\
\hline \multirow{7}{*}{ RAD51C } & Meindl et al., 2010 [46] & Family study & 6/480 families & - & - & $\mathrm{NC}$ & $\mathrm{NC}$ \\
\hline & Lilyquist et al., $2017^{\mathbb{I I}}$ [27] & Clinical testing lab & $44 / 6294(0.7 \%)$ & - & ExAC & SRR: $5.12(3.7-6.9)$ & $\mathrm{NC}$ \\
\hline & Kurian et al., $2017^{\mathbb{I}}[26]$ & Clinical testing lab & $32 / 5020(0.6 \%)$ & $72 / 64,649$ & - & OR: $4.98(3.0-8.0)$ & $\mathrm{NC}$ \\
\hline & Castera et al., $2018 \mathbb{I I}_{\text {[28] }}$ & Genetic counselling & $23 / 4309(0.5 \%)$ & - & $43 / 36,276^{\text {a }}(0.12 \%)$ & OR: 14.6 (5.3-29.5) & $\mathrm{NC}$ \\
\hline & Suszynska et al., 2019 [29] & Meta-analysis ^ & $21 / 3791(0.6 \%)$ & - & - & OR: $4.3(2.5-7.5)$ & $\mathrm{NC}$ \\
\hline & Yang et al., 2020 [10] & RAD51C families $\#$ & 125 families & - & - & RR: 7.55 (5.6-10.2) & $11 \%(15-29 \%)$ \\
\hline & Suszynska et al., 2020 [45] & Meta-analysis^^ & $149 / 23,802(0.62 \%)$ & - & $\begin{array}{c}130 / 115,475^{b} \\
(0.11 \%)\end{array}$ & OR: $5.59(4.4-7.0)$ & $\mathrm{NC}$ \\
\hline \multirow{7}{*}{$R A D 51 D$} & Loveday et al., 2011 [47] & Family study & 8/911 families & $1 / 1060(0.09 \%)$ & - & RR: 6.30 (2.8-13.8) & NC \\
\hline & Lilyquist et al., $2017^{\mathbb{I I}}$ [27] & Clinical testing lab & $11 / 5743(0.2 \%)$ & - & ExAC & $\begin{array}{l}\text { SRR: } 6.34 \\
(3.1-11.3)\end{array}$ & NC \\
\hline & Kurian et al., $2017^{\mathbb{I}}$ [26] & Clinical testing lab & $9 / 5020(0.2 \%)$ & $40 / 64,649(0.06 \%)$ & - & OR: $4.78(2.1-10.7)$ & $\mathrm{NC}$ \\
\hline & Castera et al., 2018 II [28] & Genetic counselling & $9 / 4011(0.2 \%)$ & - & $18 / 36,276^{\text {a }}(0.05 \%)$ & OR: $11.8(1.1-40)$ & $\mathrm{NC}$ \\
\hline & Suszynska et al., 2019 [29] & Meta-analysis^^ & $19 / 3258(0.6 \%)$ & - & - & OR: $11.6(5.9-23)$ & $\mathrm{NC}$ \\
\hline & Yang et al., 2020 [10] & RAD51D families $\#$ & 60 families & - & - & RR: 7.6 (5.6-10.3) & $13 \%(7-23 \%)$ \\
\hline & Suszynska et al., 2020 [45] & Meta-analysis^ & $94 / 22,787(0.45 \%)$ & - & $\begin{array}{c}72 / 120,688^{\mathrm{b}} \\
(0.06 \%)\end{array}$ & OR: 6.9 (5.1-9.4) & $\mathrm{NC}$ \\
\hline PALB2 & Yang et al., 2020 [12] & PALB2 families \# & 524 families & - & - & RR: $2.91(1.4-6.0)$ & $5 \%(2-10 \%)$ \\
\hline
\end{tabular}

Abbreviations: RR — Relative risk; OR—odds ratio; SRR—Standardized risk ratio; PVs—Pathogenic variants; -: Not available, NC: Not calculated, ${ }^{\text {a }}$ ExAC NFE non-TCGA, ${ }^{\text {b }}$ gnomAD NFE non-TCGA, ${ }^{\text {II }}$ deleterious missense changes included, ${ }^{\wedge}$ [45] Overlapped with 6 studies included in this review; ^ [29] Overlapped with 1 study included in this review; ${ }^{\#}$ Segregation analysis. Bold: highlight info. 
Table 3. Frequency of germline protein truncating variants in BRIP1, RAD51C, RAD51D, and PALB2 in EOC histotypes, in cases from population studies.

\begin{tabular}{|c|c|c|c|c|c|c|c|c|c|c|c|c|}
\hline \multirow[t]{2}{*}{ Gene } & \multirow[t]{2}{*}{ Study } & \multicolumn{7}{|c|}{$\begin{array}{c}\text { Cases } \\
\text { Carriers/Total (Frequency) }\end{array}$} & \multicolumn{2}{|c|}{$\begin{array}{c}\text { Controls } \\
\text { Carriers/Total } \\
\text { (Frequency) }\end{array}$} & \multirow{2}{*}{$\begin{array}{c}\text { Risk } \\
(95 \% \text { CI })\end{array}$} & \multirow{2}{*}{$\begin{array}{l}\text { Cumulative Risk } \\
(95 \% \mathrm{CI})^{\mathrm{a}}\end{array}$} \\
\hline & & HGS & END & $\mathrm{CCC}$ & LGS & MUC & $\begin{array}{l}\text { Mixed/ } \\
\text { Unk }{ }^{b}\end{array}$ & All EOC & Matched & $\begin{array}{c}\text { Publicly } \\
\text { Available }\end{array}$ & & \\
\hline \multirow{4}{*}{ BRIP1 } & Walsh et al., 2011 [34] & $\begin{array}{c}2 / 242 * * \\
(0.8 \%)\end{array}$ & $\begin{array}{c}1 / 23 \\
(4.3 \%)\end{array}$ & $\begin{array}{l}0 / 17 \\
(0 \%)\end{array}$ & $* *$ & - & $\begin{array}{c}1 / 78 \\
(1.3 \%)\end{array}$ & $\begin{array}{c}4 / 360 \\
(1.1 \%)\end{array}$ & - & - & $\mathrm{NC}$ & $\mathrm{NC}$ \\
\hline & Ramus et al., 2015 [11] & $\begin{array}{c}26 / 2535^{*} \\
(1.0 \%)\end{array}$ & $\begin{array}{l}0 / 65 \\
(0 \%)\end{array}$ & $\begin{array}{l}0 / 25 \\
(0 \%)\end{array}$ & $\begin{array}{l}4 / 416 \\
(0.9 \%)\end{array}$ & $\begin{array}{l}0 / 26 \\
(0 \%)\end{array}$ & $\begin{array}{l}0 / 160 \\
(0 \%)\end{array}$ & $\begin{array}{l}30 / 3227 \\
(0.92 \%)\end{array}$ & $\begin{array}{c}3 / 3444 \\
(0.09)\end{array}$ & - & $\begin{array}{l}\text { RR: } 11.2 \text { \# } \\
(3.2-34.1)\end{array}$ & $\begin{array}{c}5.8 \% \\
(3.6-9.1 \%)\end{array}$ \\
\hline & Norquist et al., 2016 [35] & $\begin{array}{c}22 / 1498 \\
(1.5 \%)\end{array}$ & $\begin{array}{c}1 / 77 \\
(1.3 \%)\end{array}$ & $\begin{array}{l}0 / 58 \\
(0 \%)\end{array}$ & $\begin{array}{l}0 / 70 \\
(0 \%)\end{array}$ & $\begin{array}{l}0 / 16 \\
(0 \%)\end{array}$ & $\begin{array}{c}3 / 196 \\
(1.5 \%)\end{array}$ & $\begin{array}{l}26 / 1915 \\
(1.36 \%)\end{array}$ & - & $\begin{array}{c}60 / 36276^{c} \\
(0.17 \%)\end{array}$ & $\begin{array}{c}\text { OR: } 6.4 \\
(3.8-10.6)\end{array}$ & $\mathrm{NC}$ \\
\hline & Total & $\begin{array}{l}50 / 4275 \\
(1.17 \%)\end{array}$ & $\begin{array}{c}2 / 165 \\
(1.21 \%)\end{array}$ & $\begin{array}{l}0 / 100 \\
(0 \%)\end{array}$ & $\begin{array}{c}4 / 486 \\
(0.82 \%)\end{array}$ & $\begin{array}{l}0 / 42 \\
(0 \%)\end{array}$ & $\begin{array}{c}4 / 434 \\
(0.9 \%)\end{array}$ & $\begin{array}{c}60 / 5502 \\
(1.1 \%)\end{array}$ & $\begin{array}{c}3 / 3444 \\
(0.09 \%)\end{array}$ & - & - & - \\
\hline \multirow{3}{*}{ RAD51C } & Song et al., $2015^{\dagger}[48]$ & $\begin{array}{c}10 / 1806^{*} \\
(0.6 \%)\end{array}$ & $\begin{array}{l}1 / 383 \\
(0.3 \%)\end{array}$ & $\begin{array}{l}1 / 225 \\
(0.4 \%)\end{array}$ & $\begin{array}{l}1 / 405 \\
(0.2 \%)\end{array}$ & $\begin{array}{l}0 / 166 \\
(0 \%)\end{array}$ & $\begin{array}{l}1 / 444 \\
(0.2 \%)\end{array}$ & $\begin{array}{l}14 / 3429 \\
(0.40 \%)\end{array}$ & $\begin{array}{c}2 / 2772 \\
(0.07 \%)\end{array}$ & - & $\begin{array}{l}\text { OR: } 5.2^{\#} \\
(1.1-24)\end{array}$ & $\begin{array}{c}5.2 \%{ }^{\mathbb{I}} \\
(1.1-22 \%)\end{array}$ \\
\hline & $\begin{array}{l}\text { Norquist et al., } 2016^{+} \\
{[35]}\end{array}$ & $\begin{array}{l}7 / 1498 \\
(0.5 \%)\end{array}$ & $\begin{array}{c}1 / 77 \\
(1.3 \%)\end{array}$ & $\begin{array}{l}0 / 58 \\
(0 \%)\end{array}$ & $\begin{array}{l}0 / 70 \\
(0 \%)\end{array}$ & $\begin{array}{l}0 / 16 \\
(0 \%)\end{array}$ & $\begin{array}{l}3 / 196 \\
(1.5 \%)\end{array}$ & $\begin{array}{l}11 / 1915 \\
(0.57 \%)\end{array}$ & - & $\begin{array}{c}39 / 36276^{\mathrm{c}} \\
(0.11 \%)\end{array}$ & $\begin{array}{l}\text { OR: } 3.4 \\
(1.5-7.6)\end{array}$ & $\mathrm{NC}$ \\
\hline & Total & $\begin{array}{l}17 / 3304 \\
(0.51 \%)\end{array}$ & $\begin{array}{c}2 / 460 \\
(0.43 \%)\end{array}$ & $\begin{array}{c}1 / 283 \\
(0.35 \%)\end{array}$ & $\begin{array}{c}1 / 475 \\
(0.21 \%)\end{array}$ & $\begin{array}{l}0 / 182 \\
(0 \%)\end{array}$ & $\begin{array}{c}/ 640 \\
(0.62 \%)\end{array}$ & $\begin{array}{l}25 / 5344 \\
(0.47 \%)\end{array}$ & $\begin{array}{c}2 / 2772 \\
(0.07 \%) \\
\end{array}$ & - & - & - \\
\hline \multirow{3}{*}{ RAD51D } & Song et al., 2015 [48] & $\begin{array}{c}9 / 1806^{*} \\
(0.5 \%)\end{array}$ & $\begin{array}{l}3 / 383 \\
(0.8 \%) \\
\end{array}$ & $\begin{array}{c}0 / 225 \\
(0 \%) \\
\end{array}$ & $\begin{array}{l}0 / 405 \\
(0 \%) \\
\end{array}$ & $\begin{array}{l}0 / 166 \\
(0 \%) \\
\end{array}$ & $\begin{array}{l}0 / 444 \\
(0 \%) \\
\end{array}$ & $\begin{array}{l}12 / 3429 \\
(0.35 \%) \\
\end{array}$ & $\begin{array}{c}1 / 2772 \\
(0.04 \%)\end{array}$ & - & $\begin{array}{l}\text { OR: 12\# } \\
(1.5-90)\end{array}$ & $\begin{array}{c}12 \% \text { II } \\
(1.5-60 \%)\end{array}$ \\
\hline & Norquist et al., 2016 [35] & $\begin{array}{l}7 / 1498 \\
(0.5 \%)\end{array}$ & $\begin{array}{c}1 / 77 \\
(1.3 \%)\end{array}$ & $\begin{array}{l}0 / 58 \\
(0 \%)\end{array}$ & $\begin{array}{l}0 / 70 \\
(0 \%)\end{array}$ & $\begin{array}{l}0 / 16 \\
(0 \%)\end{array}$ & $\begin{array}{c}3 / 196 \\
(1.5 \%)\end{array}$ & $\begin{array}{l}11 / 1915 \\
(0.57 \%)\end{array}$ & - & $\begin{array}{c}14 / 36276^{\mathrm{c}} \\
(0.04 \%)\end{array}$ & $\begin{array}{c}\text { OR: } 10.9 \\
(4.6-26.0)\end{array}$ & $\mathrm{NC}$ \\
\hline & Total & $\begin{array}{l}16 / 3304 \\
(0.48 \%)\end{array}$ & $\begin{array}{c}/ 460 \\
(0.87 \%)\end{array}$ & $\begin{array}{l}0 / 283 \\
(0 \%)\end{array}$ & $\begin{array}{l}0 / 475 \\
(0 \%)\end{array}$ & $\begin{array}{l}0 / 182 \\
(0 \%)\end{array}$ & $\begin{array}{c}3 / 640 \\
(0.47 \%)\end{array}$ & $\begin{array}{l}23 / 5344 \\
(0.43 \%)\end{array}$ & $\begin{array}{c}1 / 2772 \\
(0.04 \%)\end{array}$ & - & - & - \\
\hline \multirow{4}{*}{ PALB2 } & Ramus et al., 2015 [11] & $\begin{array}{l}6 / 2535^{*} \\
(0.24 \%)\end{array}$ & $\begin{array}{l}0 / 65 \\
(0 \%)\end{array}$ & $\begin{array}{l}1 / 25 \\
(4 \%)\end{array}$ & $\begin{array}{c}1 / 416 \\
(0.24 \%)\end{array}$ & $\begin{array}{l}0 / 26 \\
(0 \%)\end{array}$ & $\begin{array}{c}1 / 160 \\
(0.62 \%)\end{array}$ & $\begin{array}{l}9 / 3227 \\
(0.28 \%)\end{array}$ & $\begin{array}{l}3 / 3444 \\
(0.09 \%)\end{array}$ & - & $\mathrm{NC}$ & $\mathrm{NC}$ \\
\hline & Norquist et al., 2016 [35] & $\begin{array}{c}9 / 1498 \\
(0.60 \%)\end{array}$ & $\begin{array}{l}0 / 77 \\
(0 \%)\end{array}$ & $\begin{array}{c}1 / 58 \\
(1.7 \%)\end{array}$ & $\begin{array}{l}0 / 70 \\
(0 \%)\end{array}$ & $\begin{array}{l}0 / 16 \\
(0 \%)\end{array}$ & $\begin{array}{c}2 / 196 \\
(1.0 \%)\end{array}$ & $\begin{array}{l}12 / 1915 \\
(0.62 \%)\end{array}$ & - & $\begin{array}{c}39 / 36276^{c} \\
(0.10 \%)\end{array}$ & $\begin{array}{c}\text { OR: } 4.4 \\
(2.1-9.1)\end{array}$ & $\mathrm{NC}$ \\
\hline & Song et al., 2019 [54] & $\begin{array}{l}18 / 5123 \\
(0.35 \%)\end{array}$ & - & - & - & - & - & $\begin{array}{l}18 / 5123 \\
(0.35 \%)\end{array}$ & $\begin{array}{l}6 / 5202 \\
(0.12 \%)\end{array}$ & - & $\begin{array}{c}\text { OR: 3.01 \# } \\
(1.6-5.7)\end{array}$ & $\begin{array}{c}3.2 \% \\
(1.8-5.7 \%)\end{array}$ \\
\hline & Total $^{\wedge}$ & $\begin{array}{l}15 / 4033 \\
(0.37 \%)\end{array}$ & $\begin{array}{l}0 / 142 \\
(0 \%)\end{array}$ & $\begin{array}{c}2 / 83 \\
(2.4 \%)\end{array}$ & $\begin{array}{c}1 / 486 \\
(0.2 \%)\end{array}$ & $\begin{array}{l}0 / 42 \\
(0 \%)\end{array}$ & $\begin{array}{c}3 / 356 \\
(0.84 \%)\end{array}$ & $\begin{array}{l}21 / 5142 \\
(0.41 \%)\end{array}$ & $\begin{array}{c}3 / 3444 \\
(0.09 \%)\end{array}$ & - & - & \\
\hline
\end{tabular}

Abbreviations: RR—Relative risk; OR—odds ratio; SRR—Standardized risk ratio; -: Not available, NC: Not calculated due to no controls or not described, ** Frequency of serous combined, * Frequency of HGS and serous undifferentiated, ${ }^{\#}$ OR for the matched controls analysis, ${ }^{\dagger}$ Studies included one known deleterious missense variant [55], ${ }^{\text {a }}$ Risks based on all cases combined, ${ }^{\mathrm{b}}$ Mixed/Unknown includes some other histotypes, ${ }^{\mathrm{C}}$ ExAC NFE-nonTCGA, II Cumulative risks by age 70, ${ }^{\wedge}$ Total does not include Song et al., 2019 [54], study as extensive overlap with Ramus et al., 2015 [11]. Bold: highlight info. 


\subsection{Mismatch Repair (MMR) Genes}

Germline protein truncating and known deleterious missense variants in four MMR genes are associated with Lynch syndrome, which is an inherited disorder that increases the risk of many types of cancers including colon, endometrial, ovarian, pancreatic, small-bowel, and ureteric. In ovarian cancer cases from Lynch syndrome families, deleterious variants in the MMR genes were mostly seen in END and CCC cases and were more prevalent in the MSH2 and MSH6 genes [56]. This histotype-specific characteristic has been confirmed by population-based cohorts of EOC [33,34].

Germline deleterious MMR gene variants in ovarian cancer cases appear to be very rare, which may partly be because they are associated with the rare histotypes of disease [33]. Three studies have described MMR gene mutation frequencies by ovarian cancer histotypes [33-35] (Table 4).

We have combined the data across studies to provide an estimate of the frequency in these rarer histotypes (Table 4). Deleterious variants in these genes were more frequent in END and CCC cases than HGS. No variants were reported in MUC or LGS cases, although some studies combined HGS and LGS cases. Variants were more frequently found in MSH6 than the other genes, with CCC and END cases both having greater than $1 \%$ frequency.

Five other case-control studies have investigated MMR frequencies between 2000 and 7500 EOC cases, four of which included data from commercial laboratories where clinical grade testing was performed in women with hereditary cancer risk [26-28,57], while another was a meta-analysis [29]. These studies have estimated the OR for mutation carriers of these genes. For MLH1 carriers the OR was 3 (95\% CI, 1.47-6.59) [26], for MSH2 carriers, OR data from different studies ranged from 2 to 14 [26-29], and for MSH6 carriers, ranged from 2 to 9 [26-29,57]. For the MMR genes combined, the OR was estimated to be 2.3 (95\% CI, 0.83-8.2) with a cumulative risk of ovarian cancer by age 80 years of $3.7 \%(95 \%$ CI, $1.4-13 \%)$ in cases from a population-based study [33].

A Lynch syndrome prospective study had estimated the cumulative risk of ovarian cancer as a unique disease to be $10 \%$ (95\% CI 4.8-15.4\%) for MLH1 carriers by the age of 75 years, $17 \%$ (95\% CI 5.7-28.0\%) for MSH2 carriers, and 13\% (95\% CI 0.1-31.2\%) for MSH6 carriers [13]. The risk in the different EOC histotypes was not reported [13], although it is known that they are mostly seen in END and CCC cases.

The cumulative risk of endometrial cancer by the age of 75 years was estimated to be $43 \%$ (95\% CI $33.1-52.3 \%$ ), 57\% (95\% CI 41.8-71.6\%), and 46\% (95\% CI 27.3-65.0\%) for MLH1, MSH2, and MSH6 carriers, respectively [13]. Due to the increased risk of both ovarian and endometrial cancer in women with Lynch syndrome, discussion about RRSO with hysterectomy is recommended. MMR carriers from these Lynch syndrome families were also reported to have an increased risk of colorectal, upper gastrointestinal, urinary tract, prostate, and brain cancers [13].

\section{Missense Variants in Confirmed Susceptibility Genes}

Protein truncating variants have been classified as deleterious, however a relatively large number of missense variants, including some rare predicted pathogenic missense variants have also been identified in these genes. Determining if any of these missense variants are also pathogenic is important for patient management and risk prediction in families.

\subsection{BRCA1/2 and MMR Genes}

Over many years, due to efforts of commercial labs, independent research groups, and large consortia, many missense variants of unknown significance (VUS) or unclassified variants (UV) have been reclassified as either pathogenic or benign. However, there are still many variants that remain unclassified. Large-scale consortia efforts, such as the Evidence-based Network for the Interpretation of Germline Mutant Alleles (ENIGMA) are in the process of investigating and classifying these variants in the $B R C A 1 / 2$ genes and the interpretation of the results of genetic testing for reporting and counselling [58]. 
Table 4. Frequency of germline pathogenic variants in the mismatch repair (MMR) genes in EOC histotypes, in cases from population studies.

\begin{tabular}{|c|c|c|c|c|c|c|c|c|c|}
\hline \multirow{2}{*}{ Gene } & \multirow{2}{*}{ Study } & \multicolumn{7}{|c|}{ Cases Carriers/Total (Frequency) } & \multirow{2}{*}{$\begin{array}{c}\begin{array}{c}\text { Controls Carriers/Total } \\
\text { (Frequency) }\end{array} \\
\text { Matched } \\
\end{array}$} \\
\hline & & HGS & END & $\mathrm{CCC}$ & LGS & MUC & Mixed/Unk ${ }^{a}$ & All EOC & \\
\hline \multirow{3}{*}{ MLH1 } & Song et al., 2014 [33] & $\begin{array}{c}0 / 1105 * \\
(0 \%)\end{array}$ & $\begin{array}{c}0 / 322 \\
(0 \%)\end{array}$ & $\begin{array}{c}1 / 192 \\
(0.52 \%)\end{array}$ & $\begin{array}{c}0 / 172 \\
(0 \%)\end{array}$ & $\begin{array}{l}0 / 157 \\
(0 \%)\end{array}$ & $\begin{array}{l}0 / 274 \\
(0 \%)\end{array}$ & $\begin{array}{c}1 / 2222 \\
(0.04 \%)\end{array}$ & $\begin{array}{l}2 / 1528 \\
(0.13 \%)\end{array}$ \\
\hline & Norquist et al., 2016 [35] & $\begin{array}{c}0 / 1498 \\
(0 \%)\end{array}$ & $\begin{array}{l}0 / 77 \\
(0 \%)\end{array}$ & $\begin{array}{l}0 / 58 \\
(0 \%)\end{array}$ & $\begin{array}{l}0 / 70 \\
(0 \%)\end{array}$ & $\begin{array}{l}0 / 16 \\
(0 \%)\end{array}$ & $\begin{array}{l}1 / 196^{\mathrm{b}} \\
(0.51 \%)\end{array}$ & $\begin{array}{c}1 / 1915 \\
(0.05 \%)\end{array}$ & - \\
\hline & Total & $\begin{array}{c}0 / 2603 \\
(0 \%)\end{array}$ & $\begin{array}{l}0 / 399 \\
(0 \%)\end{array}$ & $\begin{array}{c}1 / 250 \\
(0.40 \%)\end{array}$ & $\begin{array}{l}0 / 242 \\
(0 \%)\end{array}$ & $\begin{array}{l}0 / 173 \\
(0 \%)\end{array}$ & $\begin{array}{c}1 / 470 \\
(0.21 \%)\end{array}$ & $\begin{array}{c}2 / 4137 \\
(0.04 \%)\end{array}$ & $\begin{array}{c}2 / 1528 \\
(0.13 \%)\end{array}$ \\
\hline \multirow{2}{*}{ MSH2 } & Song et al., 2014 [33] & $\begin{array}{l}1 / 1105^{*} \\
(0.09 \%)\end{array}$ & $\begin{array}{l}0 / 322 \\
(0 \%)\end{array}$ & $\begin{array}{c}1 / 192 \\
(0.52 \%)\end{array}$ & $\begin{array}{l}0 / 172 \\
(0 \%)\end{array}$ & $\begin{array}{l}0 / 157 \\
(0 \%)\end{array}$ & $\begin{array}{c}0 / 274 \\
(0 \%)\end{array}$ & $\begin{array}{l}2 / 2222 \\
(0.09 \%)\end{array}$ & $\begin{array}{c}0 / 1528 \\
(0 \%)\end{array}$ \\
\hline & Total & $\begin{array}{c}1 / 1105 \\
(0.09 \%)\end{array}$ & $\begin{array}{l}0 / 322 \\
(0 \%)\end{array}$ & $\begin{array}{c}1 / 192 \\
(0.52 \%)\end{array}$ & $\begin{array}{l}0 / 172 \\
(0 \%)\end{array}$ & $\begin{array}{l}0 / 157 \\
(0 \%)\end{array}$ & $\begin{array}{l}0 / 274 \\
(0 \%)\end{array}$ & $\begin{array}{c}2 / 2222 \\
(0.09 \%)\end{array}$ & $\begin{array}{c}0 / 1528 \\
(0 \%)\end{array}$ \\
\hline \multirow{4}{*}{ MSH6 } & Walsh et al., 2011 [34] & $\begin{array}{c}0 / 242 * * \\
(0 \%)\end{array}$ & $\begin{array}{c}2 / 23 \\
(8.7 \%)\end{array}$ & $\begin{array}{l}0 / 17 \\
(0 \%)\end{array}$ & $* *$ & - & $\begin{array}{l}0 / 78 \\
(0 \%)\end{array}$ & $\begin{array}{c}2 / 360 \\
(0.55 \%) \\
\end{array}$ & - \\
\hline & Song et al., 2014 [33] & $\begin{array}{l}4 / 1105^{*} \\
(0.36 \%)\end{array}$ & $\begin{array}{c}2 / 322 \\
(0.62 \%)\end{array}$ & $\begin{array}{c}3 / 192 \\
(1.56 \%)\end{array}$ & $\begin{array}{l}0 / 172 \\
(0 \%)\end{array}$ & $\begin{array}{l}0 / 157 \\
(0 \%)\end{array}$ & $\begin{array}{l}0 / 274 \\
(0 \%)\end{array}$ & $\begin{array}{l}9 / 2222 \\
(0.40 \%)\end{array}$ & $\begin{array}{l}1 / 1528 \\
(0.06 \%)\end{array}$ \\
\hline & Norquist et al., 2016 [35] & $\begin{array}{l}1 / 1498 \\
(0.06 \%)\end{array}$ & $\begin{array}{c}2 / 77 \\
(2.59 \%)\end{array}$ & $\begin{array}{l}0 / 58 \\
(0 \%)\end{array}$ & $\begin{array}{l}0 / 70 \\
(0 \%)\end{array}$ & $\begin{array}{l}0 / 16 \\
(0 \%)\end{array}$ & $\begin{array}{l}0 / 196 \\
(0 \%)\end{array}$ & $\begin{array}{c}/ 1915 \\
(0.15 \%)\end{array}$ & - \\
\hline & Total & $\begin{array}{c}5 / 2845 \\
(0.17 \%) \\
\end{array}$ & $\begin{array}{c}6 / 422 \\
(1.42 \%)\end{array}$ & $\begin{array}{c}3 / 267 \\
(1.12 \%)\end{array}$ & $\begin{array}{l}0 / 242 \\
(0 \%)\end{array}$ & $\begin{array}{l}0 / 173 \\
(0 \%)\end{array}$ & $\begin{array}{l}0 / 548 \\
(0 \%)\end{array}$ & $\begin{array}{l}14 / 4497 \\
(0.31 \%)\end{array}$ & $\begin{array}{l}1 / 1528 \\
(0.06 \%)\end{array}$ \\
\hline \multirow{3}{*}{ PMS2 } & Song et al., 2014 [33] & $\begin{array}{c}0 / 1105 * \\
(0 \%)\end{array}$ & $\begin{array}{c}1 / 322 \\
(0.31 \%)\end{array}$ & $\begin{array}{c}0 / 192 \\
(0 \%)\end{array}$ & $\begin{array}{l}0 / 172 \\
(0 \%)\end{array}$ & $\begin{array}{c}0 / 157 \\
(0.6 \%)\end{array}$ & $\begin{array}{l}0 / 274 \\
(0 \%)\end{array}$ & $\begin{array}{c}1 / 2222 \\
(0.04 \%)\end{array}$ & $\begin{array}{c}0 / 1528 \\
(0 \%)\end{array}$ \\
\hline & Norquist et al., 2016 [35] & $\begin{array}{l}4 / 1498 \\
(0.3 \%)\end{array}$ & $\begin{array}{l}0 / 77 \\
(0 \%)\end{array}$ & $\begin{array}{l}0 / 58 \\
(0 \%)\end{array}$ & $\begin{array}{l}0 / 70 \\
(0 \%)\end{array}$ & $\begin{array}{l}0 / 16 \\
(0 \%)\end{array}$ & $\begin{array}{l}0 / 196 \\
(0 \%)\end{array}$ & $\begin{array}{c}/ 1915 \\
(0.20 \%)\end{array}$ & - \\
\hline & Total & $\begin{array}{c}4 / 2603 \\
(0.15 \%)\end{array}$ & $\begin{array}{c}1 / 399 \\
(0.25 \%)\end{array}$ & $\begin{array}{l}0 / 250 \\
(0 \%)\end{array}$ & $\begin{array}{l}0 / 242 \\
(0 \%)\end{array}$ & $\begin{array}{l}0 / 173 \\
(0 \%)\end{array}$ & $\begin{array}{l}0 / 470 \\
(0 \%)\end{array}$ & $\begin{array}{l}5 / 4137 \\
(0.12 \%)\end{array}$ & $\begin{array}{c}0 / 1528 \\
(0 \%)\end{array}$ \\
\hline All 4 genes & Total $^{\$}$ & $\begin{array}{l}10 / 2845 \\
(0.35 \%)\end{array}$ & $\begin{array}{c}7 / 422 \\
(1.65 \%)\end{array}$ & $\begin{array}{c}5 / 267 \\
(1.87 \%)\end{array}$ & $\begin{array}{l}0 / 242 \\
(0 \%)\end{array}$ & $\begin{array}{l}0 / 173 \\
(0 \%)\end{array}$ & $\begin{array}{c}1 / 548 \\
(0.18 \%)\end{array}$ & $\begin{array}{l}23 / 4497 \\
(0.51 \%)\end{array}$ & $\begin{array}{c}3 / 1528 \\
(0.19 \%)\end{array}$ \\
\hline
\end{tabular}

-: Not available, ${ }^{* *}$ serous combined, ${ }^{*}$ HGS and serous undifferentiated, ${ }^{a}$ Mixed/Unknown includes some other histotypes, ${ }^{b}$ MLH1 variant found in carcinosarcoma, ${ }^{\$}$ Totals did not include overlapped studies. Bold: highlight info. 
To date, the National Institutes of Health (NIH) repository, ClinVar, has reported approximately 7000 pathogenic or likely pathogenic variants and 7400 VUS in BRCA1/2 genes (mostly missense changes, but also in-frame deletions and insertions, and intronic and exonic variants that may affect splicing efficiency) [59]. The same clinical challenge occurs in patients with variants in the MMR genes, approximately $30 \%$ of all variants are classified as VUS [60].

Individual studies may include analysis of missense variants using bioinformatic tools such as SIFT [61], PolyPhen-2 [62], and Provean [63] to predict the functional effect of variants. Association between predicted deleterious rare missense variants and ovarian cancer risk can be assessed using burden tests, such as the rare admixture maximum likelihood test (RAML), that accounts for differences in risk of each associated variant [64]. Song et al. have performed this type of analysis in 131 predicted deleterious missense variants in BRCA1/2 and the MMR genes (MLH1, MSH2, MSH6, and PMS2) and found little evidence for ovarian cancer risk association between these variants in any of the six genes [33].

\subsection{BRIP1, RAD51C/D and PALB2}

Missense variants in the moderate-risk genes recently confirmed as susceptibility genes in ovarian cancer need further investigation to determine if any are likely to be pathogenic. Some studies have included predicted deleterious missense variants in estimating mutation frequencies.

Ramus et al. used SIFT, PolyPhen-2, and Provean scores to predict if uncommon (minor allele frequency $(\mathrm{MAF})<1 \%$ ) and rare $(\mathrm{MAF}<0.1 \%)$ missense variants were potentially deleterious and identified 35 for BRIP1 and 26 in PALB2. They performed the RAML burden test and found an increased risk association for uncommon and rare missense variants in BRIP1, but not for PALB2 [11,54].

In $R A D 51 C / D$, Song et al. performed the RAML test for 28 rare predicted deleterious missense variants (12 in $R A D 51 C$ and 16 in $R A D 51 D$ ) [48]. Evidence for an association of rare predicted deleterious missense variants with an increased risk of ovarian cancer was observed in both genes [48] (Table 5).

The data from the RAML burden test suggest that some of the predicted deleterious missense variants in BRIP1, RAD51C, and RAD51D likely increase disease risk, but it does not indicate how many or which ones. There is no evidence for specific missense variants. 
Table 5. Predicted deleterious missense variants in known moderate risk genes for EOC.

\begin{tabular}{|c|c|c|c|c|}
\hline Genes & Study & Cases Del Missense/Total (Frequency) & Predicted Deleterious & Evidence of Risk from RAML \\
\hline \multirow[t]{2}{*}{ BRIP1 } & Ramus et al., 2015 [11] & $\begin{array}{c}35 / 3227^{\mathrm{a}} \\
(1.1 \%) \\
\end{array}$ & SIFT, PolyPhen-2 and Provean & $\begin{array}{c}\text { Yes } \\
\text { (All EOC, but stronger in HGS) }\end{array}$ \\
\hline & Suszynska et al., 2020 [45] & $2 * / 22494$ & Described in ClinVar & - \\
\hline \multirow{4}{*}{ RAD51C } & Song et al., 2015 [11] & $\begin{array}{l}12 / 3429^{b} \\
(0.32 \%)\end{array}$ & SIFT, PolyPhen-2 and Provean & $\begin{array}{c}\text { Yes } \\
\text { (All EOC, but stronger in HGS) }\end{array}$ \\
\hline & Lilyquist et al., 2017 [27] & $2 \$ / 6294$ & Described in ClinVar & - \\
\hline & Norquist et al., 2016 [35] & $1^{\# / 1915^{c}}$ & Functional assay [65] & - \\
\hline & Suszynska et al., 2020 [45] & $3^{\wedge} / 22,494$ & Described in ClinVar & - \\
\hline \multirow{2}{*}{ RAD51D } & Song et al., 2015 [11] & $\begin{array}{l}16 / 3429 \\
(0.46 \%)\end{array}$ & SIFT, PolyPhen-2 and Provean & $\begin{array}{c}\text { Yes } \\
\text { (Only in HGS) }\end{array}$ \\
\hline & Suszynska et al., 2020 [45] & $1 \mathbb{I} / 22,494$ & Described in ClinVar & - \\
\hline \multirow{2}{*}{ PALB2 } & Ramus et al., 2015 [11] & $\begin{array}{l}26 / 3227 \\
(0.80 \%) \\
\end{array}$ & SIFT, PolyPhen-2 and Provean & No \\
\hline & Song et al., 2019 [54] & $\begin{array}{c}40 / 5123^{\mathrm{a}} \\
(0.78 \%)\end{array}$ & SIFT, PolyPhen-2 and Provean & No \\
\hline
\end{tabular}

-: Not available, ${ }^{\mathrm{a}}$ All variants reported in $\mathrm{HGS},{ }^{\mathrm{b}}$ One variant found in LGS - (histotype not available for the other variants), ${ }^{\mathrm{c}}$ Variant found in a serous carcinoma. ClinVar reported variants: * p.Gln169 = and p.Ala349Pro; ${ }^{\$}$ p.C135Y and p.L138F; ${ }^{*}$ p.Q143R; ^ p.Cys135Tyr, p.Cys135Ser and p.Leu138Phe; ${ }^{\mathbb{I I}}$ p.Ser207Leu. 


\section{Deleterious Variants in Other Proposed Susceptibility Genes}

\subsection{FANCM}

FANCM is part of the Fanconi anemia group together with BRCA2, BRIP1 and PALB2 (also known as FANCD1, FANCJ, and FANCN). A case-control study was performed by Dicks et al. where FANCM targeted sequencing was performed in 3107 HGS cases, 1491 cases of other histotypes, and 3368 unaffected matched controls [66]. A significantly higher frequency of protein truncating variants was found in the HGS cases compared to the controls $(p 0.008)$ and no evidence of association was observed with other histotypes ( $p$ 0.82) [66]. The relative risk for HGS was estimated to be 2.5 (95\% CI 1.3-5) with lifetime average risk of 3.8\% (80\% CI 2.2-4.5\%) [66]. Lifetime risk was increased when known ovarian cancer risk factors such as common risk alleles and lifestyle factors were taken into account (4.6\% (80\% CI 3.1-7.0\%) and 5.2\% (80\% CI 3.4-7.8\%), respectively) [66]. Additionally, the RAML test was performed for 243 uncommon predicted deleterious missense variants (effect given by at least two of the three function prediction programs Polyphen-2, Provean, and SIFT), but there was no difference in the frequency of these variants in cases compared to controls [66].

Although this study had shown evidence of association between germline protein truncating variants in FANCM and moderate increase in risk of HGS ovarian cancer, larger and prospective studies are still needed to confirm this.

\subsection{ATM}

ATM is a candidate ovarian cancer susceptibility gene due to its role in breast [67] and pancreatic cancer [68]. Germline heterozygous pathogenic ATM variants are associated with fivefold higher risk of breast cancer by the age of 50 years [67] and some rare variants have been shown to have penetrance as high as the BRCA2 gene [69].

In ovarian cancer, case-control studies mainly enriched for a family history of breast or ovarian cancer, have suggested that pathogenic or likely pathogenic variants in ATM might be associated with moderate increased risk (OR 1.69, 95\% CI 1.2-2.4 [26]; Standardized risk ratio (SRR) 2.25, 95\% CI 1.7-3 [27]; OR 1.97, 95\% CI 1.3-3 [29]; OR 2.4, 95\% CI 1.2-4.7 [35]; OR 2.85, 95\% CI 1.3-6.3 [57]), however the cumulative lifetime risk has been estimated to be lower than $3 \%$.

\subsection{BARD1 and NBN}

The BARD1 and NBN genes were included in breast/ovarian cancer genetic panel tests, due to their breast cancer risk, despite the ovarian cancer risk for deleterious variants in these genes being unknown. The BARD1 encoded protein interacts closely with the BRCA1 protein due to sharing the N-terminal RING finger and the BRCA1 C-terminal domains. Interaction between these genes affects double-strand break repair and apoptosis suggesting that this protein may play an important role in BRCA1 tumour suppression [70]. Consequently, BARD1 was considered a potential candidate susceptibility gene for ovarian cancer. However, Ramus et al. found no significant differences in $B A R D 1$ deleterious variants frequency in a cohort of $\sim 3200$ ovarian cancer cases compared with $\sim 3400$ matched-controls ( $p$ 0.39) [11]. Several additional case-control studies confirmed that no evidence of association was observed between ovarian cancer risk and pathogenic variants in BARD1 (OR 0.59, 95\% CI 0.21-1.68 [26]; SSR 1.28, 95\% CI 0.55-2.51 [27]; OR 1.4, 95\% CI 0.7-2.9 [29]). A moderate increase in breast cancer risk has been suggested but more evidence is needed to validate these findings [70,71].

The NBN encoded protein interacts with MRE11A and RAD50 encoded proteins as arge complex, which interacts with the protein produced by the ATM gene. These combined proteins have an important role in identifying broken strands of DNA and repairing it. Due to its essential function in the DNA repair pathway and because this gene is commercially available in gene testing panels for ovarian cancer, case-controls studies have examined if they are susceptibility genes. However, most studies have not found a higher frequency of pathogenic variants in NBN, MRE11A, or RAD50 in 
cases compared to controls (NBN: $p 0.61$ [11] and 0.09 [35]; MRE11A: $p$ 1.0 [35]; RAD50: $p 0.63$ [35]) and therefore no evidence of association with risk was observed in any of these 3 genes.

\subsection{CHECK2}

Germline pathogenic variants in CHECK2 (a cell cycle checkpoint regulator) are associated with an increased risk of breast cancer [72] and CHECK2 was proposed to be a candidate gene for ovarian cancer risk on that basis. A prospective study had shown that in estrogen receptor-positive breast cancer, CHEK2*1100delC heterozygosity was associated with increased risk of early death, breast cancer-specific death, and risk of a second breast cancer [72]. However, numerous retrospective case-control studies did not find an association between pathogenic variants in CHECK2 and an increased risk of ovarian cancer $[26,27,35]$.

\subsection{Other Genes}

TP53 was suggested as a potential candidate susceptibility gene for ovarian cancer from a case only study [34] and from a whole-exome sequencing analysis where protein truncating variants were identified at a greater frequency in ovarian cancer cases compared to publicly available controls (OR 18.5; 95\% CI, 2.6-808.1) [34,57]. This gene has not been validated in other targeted sequencing and WES studies [26,35].

Other candidate genes selected for targeted sequencing validation in cases and controls have shown weak evidence of association with ovarian cancer risk for protein truncating variants in $P O L K$, SLX4 (also known as FANCP), and FBXO10, but further studies are required to confirm this [54]. Several Fanconi Anaemia genes (FANCA, FANCB, FANCC, FANCD2, FANCE, FANCG, FANCI, and FANCL) have been tested in these case-control studies but a risk association has not been detected [54]. A large number of other genes have been examined and not been shown to be ovarian cancer risk genes $[48,57,66]$.

\section{Discussion}

We have presented a comprehensive review of the contribution of rare genetic variants to ovarian cancer, with a focus on the relationship between genetic variation and ovarian cancer histotypes, using data mostly only presented as supplementary in the original papers.

For $B R C A 1 / 2$, deleterious germline variants are more common in HGS ( $8 \%$ for $B R C A 1$ and $6 \%$ for $B R C A 2$ ), but there is a significant frequency of variants in END ( $3 \%$ for both genes), CCC ( $3 \%$ for $B R C A 1$ and $1 \%$ for $B R C A 2$ ), and LGS ( $3 \%$ for $B R C A 1$ and $2 \%$ for $B R C A 2$ ) patients. Consequently, the current guidelines recommend $B R C A 1 / 2$ testing in all non-mucinous ovarian cancer cases. For the MMR genes, the frequency of deleterious germline variants is higher in END and CCC patients, with variants in MSH6 being more common than variants in MLH1, MSH2, and PMS2 (greater than 1\% in MSH6 and approximately or less than $0.5 \%$ in MLH1/MSH2/PMS2). For BRIP1, the frequency of protein truncating variants seems to be similar for HGS and END (approximately 1.2\%), but slightly lower for LGS cases $(0.8 \%)$. For RAD51C, a slightly higher frequency of variants in the HGS cases is observed $(0.5 \%)$, and frequencies range from 0.2 to $0.4 \%$ in END, CCC, and LGS. No variants in MUC were found. In contrast, a higher frequency of protein truncating variants in END cases is seen for RAD51D $(0.9 \%)$, compared to the HGS cases $(0.5 \%)$. No variants were detected in CCC, LGS, or MUC. A higher frequency of protein truncating variants in CCC is seen in PALB2, compared to $0.4 \%$ in HGS and $0.2 \%$ in LGS. However, larger numbers of the non-HGS cases are needed to confirm these frequencies especially in the moderate risk genes that have been recently confirmed as susceptibility genes for ovarian cancer.

The role of individual missense variants in the known ovarian cancer genes is not yet known. Work by the ENIGMA consortium is underway to identify deleterious missense variants in $B R C A 1$ and $B R C A 2$, and ongoing efforts will be required for other genes [58].

The frequency of large genomic alternations (insertions, deletions, and rearrangements) have been examined in BRCA1/2 and the MMR genes [17,73]. These types of changes make up $8-40 \%$ of $B R C A 1$ mutations, depending on the population [17]. We do not yet know how frequent they are in the BRIP1, 
$R A D 51 C, R A D 51 D$, and PALB2 genes, and this could affect prevalence estimates. These types of changes cannot be detected with the methods currently used for targeted sequencing in clinical panel testing.

As outcomes in ovarian cancer are linked to its stage at diagnosis, the ability to identify women at risk earlier or before diagnosis has important clinical implications. The use of multigene panel testing has allowed women to be tested for multiple genetic variants associated with increased cancer risk, enabling personalised risk estimates to be developed [74,75]. While screening for ovarian cancer has not been shown to reduce mortality, RRSO can be offered to women at a sufficiently high risk of developing ovarian cancer $[4,5]$.

There has been some debate as to what level of lifetime cancer risk justifies prophylactic surgical intervention. Traditionally, a threshold of greater than $10 \%$ lifetime ovarian cancer risk was used, allowing for RRSO in women carrying $B R C A 1, B R C A 2$, or mismatch repair gene mutations, which confer lifetime ovarian cancer risk well in excess of $10 \%$ [76]. The more recent discovery of moderate penetrance genes has prompted formal studies into risk thresholds for invention, and recent analyses have suggested that offering RRSO to women with a lifetime risk as low as $4-5 \%$ can be cost-effective [50]. On that basis, women carrying variants in BRIP1, RAD51C, and RAD51D may also be offered RRSO [77,78].

The best approach to managing patients with genetic variants that do not sufficiently increase ovarian cancer risk to justify RRSO but increase risk to higher than that of the general population is not yet known. While it is plausible that targeting these women for screening may allow for detection of cancers at an earlier stage, this has not been studied. Studies investigating the impact of ovarian cancer screening using ultrasonographic and biochemical methods in both the general population, and high-risk groups (greater than $10 \%$ risk on the basis of family history or presence of genetic variants) have been associated with stage-shift, but have not been shown to significantly reduce mortality [5,79].

Genetic testing is fraught with ethical, legal, and psychosocial implications for patients, and the rate of advancement in our understanding of cancer genetics often outstrips our ability to use information in the clinic $[80,81]$. It is important to note that genetic susceptibility to EOC cancer does not exist in isolation, and variants conferring increased ovarian cancer risk also often increase the risk of developing other cancers-most notably breast cancer in BRCA1 and BRCA2, and colon cancer in Lynch syndrome. Furthermore, breast and ovarian cancer genes are often combined into a single panel test, potentially uncovering variants in genes for which pathogenicity is disputed. As an example, PALB2 is associated with a lifetime breast cancer risk of $53 \%$, but also lifetime risks of $5 \%$ and $2-3 \%$ for ovarian and pancreatic malignancies, respectively [12]. While a 53\% lifetime breast cancer risk is clearly clinically actionable, the risk of ovarian and pancreatic cancers associated with PALB2 exists in an area of clinical uncertainty. The opposite is seen for deleterious variants in BRIP1, with an increased risk of ovarian cancer but no increase in breast cancer risk. For these reasons, all women undergoing genetic testing for familial susceptibility to ovarian cancer should receive appropriate pre- and post-test counselling, and follow-up with relevant clinical services. Genetic information should be applied cautiously in the clinic, after careful evaluation of the available literature, as some genes included on so called "ovarian cancer" panel tests have now been shown not to increase ovarian cancer risk $[82,83]$ (Table 6). The risks of ovarian and breast cancer for genes in the double-strand DNA break repair pathway are shown in Figure 1.

Current testing guidelines do not recommend population-based genetic testing for ovarian cancer, however population-based testing approaches may be effective in groups with a small number of common founder mutations such as the three BRCA1/2 variants in Ashkenazi Jews [86,87]. With the decreasing costs of sequencing methods, these approaches may also become cost effective in other populations. Population-based genetic testing has many issues, including cultural and psychosocial, that need to be investigated [87]. The majority of the genetic studies in ovarian cancer have been performed in white populations, and work on other ethnic groups is ongoing [88]. 
Table 6. Summary of the frequency of deleterious variants by EOC histotype for each homologous recombination (HR) gene and the MMR genes, and a comparison of risk and clinical management for ovarian and breast cancer patients.

\begin{tabular}{|c|c|c|c|c|c|c|c|c|c|c|c|}
\hline \multirow{2}{*}{ Genes } & \multicolumn{5}{|c|}{ Frequency (\%) } & \multicolumn{2}{|c|}{ Risk Est. ^ } & \multicolumn{2}{|c|}{ Risk Level } & \multicolumn{2}{|c|}{ Clinical Management $^{\$}$} \\
\hline & HGS & END & $\mathrm{CCC}$ & LGS & MUC & EOC & BC & EOC & BC & EOC & BC \\
\hline$B R C A 1$ & 7.8 & 2.9 & 3.6 & 3.7 & $<1$ & 60 & $72 \% *$ & Very high & Very high & \multirow{2}{*}{ RRSO age 35 to 45 PARPi } & \multirow{2}{*}{ RRM age 25 to 40} \\
\hline$B R C A 2$ & 5.9 & 2.9 & $<1$ & 2 & $<1$ & 17 & $69 \% *$ & High & Very high & & \\
\hline$M M R$ & $<1$ & 1.6 & 1.9 & 0 & 0 & 2.3 & - & Mod & None & RRSO with hysterectomy for LS & No increased risk \\
\hline BRIP1 & 1.2 & 1.2 & 0 & $<1$ & 0 & 11.2 & - & Mod & None & \multirow{3}{*}{$\begin{array}{l}\text { RRSO age } 45 \text { to } 50 \\
\text { no consensus }\end{array}$} & \multirow{3}{*}{ Insufficient evidence } \\
\hline RAD51C & $<1$ & $<1$ & $<1$ & $<1$ & 0 & 5.2 & 1.9 & Mod & None & & \\
\hline RAD51D & $<1$ & $<1$ & 0 & 0 & 0 & 12 & 1.8 & Mod & None & & \\
\hline PALB2 & $<1$ & 0 & 2.4 & $<1$ & 0 & 3.0 & 7.2 & Low & Mod & Insufficient evidence & $\begin{array}{c}\text { Annual mammography/breast MRI } \\
\text { age } 30 \\
\text { no consensus }\end{array}$ \\
\hline TP53 & \multicolumn{5}{|c|}{ Insufficient data } & Insuf & Insuf & Low & Mod & Insufficient evidence & Insufficient evidence \\
\hline CHEK2 & \multicolumn{5}{|c|}{ No increased risk } & - & 3.0 & None & Low & \multirow{3}{*}{\multicolumn{2}{|c|}{$\begin{array}{c}\text { Annual mammography/breast MRI } \\
\text { age } 40 \\
\text { no consensus }\end{array}$}} \\
\hline$A T M$ & \multicolumn{5}{|c|}{ No increased risk } & - & 2.8 & None & Low & & \\
\hline NBN & \multicolumn{5}{|c|}{ No increased risk } & - & 2.7 & None & Low & & \\
\hline RAD50 & \multicolumn{5}{|c|}{ No increased risk } & - & Insuf & None & Low & \multirow{2}{*}{ No increased risk } & \multirow{2}{*}{ Insufficient evidence } \\
\hline MRE11A & & & crease & & & - & Insuf & None & Low & & \\
\hline
\end{tabular}

Abbreviations: BC—breast cancer; RRM—Risk-reducing mastectomy; LS—Lynch Syndrome; MRI—Magnetic Resonance Imaging; Insuf—Insufficient. ^ From Tables 1, 3 and 4. EOC $B R C A 1 / 2$ from ref [33], MMR from ref [33], BRIP1 from ref [11], RAD51C/D from ref [48], PALB2 from ref [54]. BC BRCA1/2 from ref [9], RAD51C/D from ref [10], PALB2 from ref [12], CHEK2/ATM/NBN from ref [84]. ${ }^{\$} B R C A 1 / 2$ from refs [39,85], MMR from ref [13], BRIP1/RAD51C/D from ref [49], PALB2/CHEK2/ATM/NBN from refs [77,84]. * Cumulative risk data by the age of 80 . 

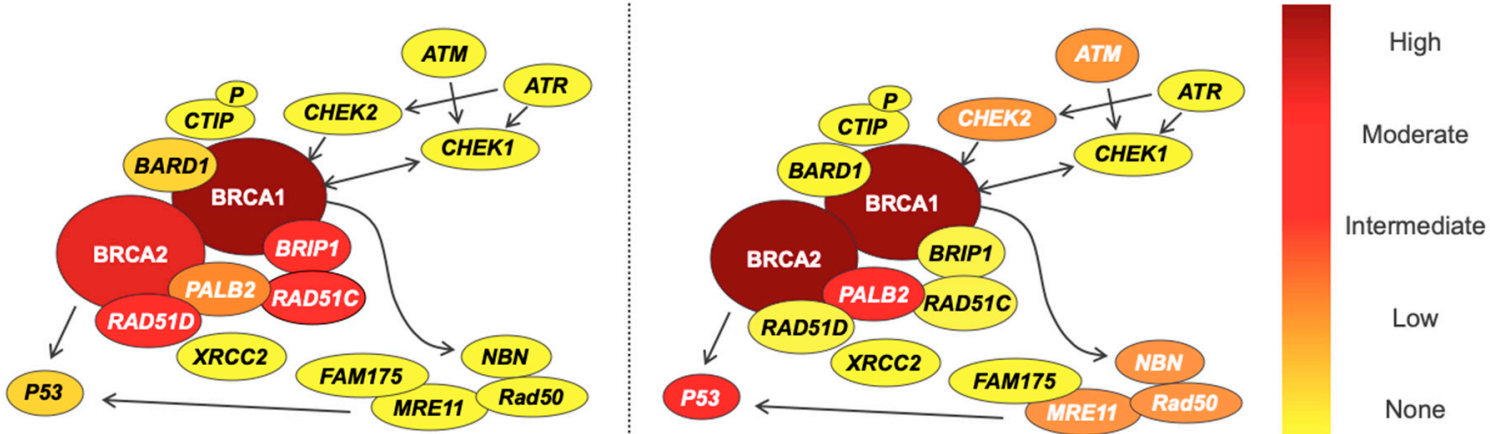

Figure 1. Susceptibility genes present in the double-strand DNA break repair pathway for ovarian and breast cancer and its different correspondent risks levels in each disease.

Identifying ovarian cancer genes has translated to novel therapeutic options for patients. PARP inhibitors, which target double-stranded DNA repair in cells with deficient homologous recombination, including cells with dysfunctional BRCA DNA repair pathways, have been shown to improve progression free survival (PFS) in serous and endometrioid ovarian cancers [89-92]. The impact of PARP inhibitors on PFS is most dramatic in patients with mutations in BRCA1 and BRCA2, but can also benefit women without BRCA mutations if they are found to have deficiencies in homologous recombination, such as variants in the DNA repair genes discussed above [92]. However, improvements in PFS do not necessarily translate to improved overall survival, and the impact of PARP inhibitors on the overall survival endpoint has not yet been reported [92].

To date, ovarian cancer has largely been treated as a single entity. However, as outlined above, the five main histotypes of epithelial ovarian cancer appear to be characterised by distinct genetic mutations. The tumours also have different molecular profiles, and patients have different treatment responses [93]. Therefore, it has become increasingly clear that ovarian cancer represents not just a single disease but encompasses a number of distinct cancers. Most of our understanding of ovarian cancer genes relates to high-grade serous tumours, as they represent the majority of ovarian cancers. Identifying genetic variants contributing to non-serous ovarian cancers has posed an ongoing challenge for researchers, as finding rare variants for rare cancers requires studies with sample sizes that are not yet feasible.

Despite significant advancements in our understanding of the genetic epidemiology of ovarian cancer, the known ovarian cancer risk variants explain less than half of the excess familial risk for ovarian cancer [8]. Efforts to identify novel genetic variants associated with ovarian cancer are ongoing. The discovery of new genetic variants should be accompanied by efforts to accurately quantify the exact magnitude of increased risk associated with that variant. Case-control studies, the most commonly used study design in gene discovery, are only able to generate relative risks, from which absolute risks are extrapolated.

Prospective population-based studies, which have been used to provide gold-standard estimates of lifetime cancer risk in $B R C A 1, B R C A 2$, and the mismatch repair genes, would provide more accurate risk estimates for moderate penetrance ovarian cancer genes, but are costly, and require long-term investment. Segregation analysis from large international consortia has been used to calculate ovarian cancer risks in RAD51C, RAD51D, and PALB2, and could be applied to other moderate risk ovarian cancer genes $[10,12]$.

Genetic association studies, by their design, are unable to establish a causal relationship between germline variant status and cancer phenotype. The discovery of novel ovarian cancer risk genes should be followed-up by further efforts to characterise the functional mechanisms by which they contribute to cancer development and identify potential therapeutic targets. 


\section{Conclusions}

Ovarian cancer is the most lethal gynaecological malignancy, and an improved understanding of the contribution of rare genetic variants to the development of ovarian cancer allows for better clinical management of at-risk women. Based on the best available evidence, variants in BRCA1, BRCA2, $B R I P 1, R A D 51 C, R A D 51 D$, and the mismatch repair genes confer ovarian cancer risks that warrant the consideration of risk-reducing surgery. The best approach for managing women with deleterious variants in ovarian cancer genes that do not warrant prophylactic surgery requires further investigation.

Funding: M.P. is funded by UNSW Sydney, the Yarrow Family and the Australian Government Research Training Program Scholarship. This work was funded by Cancer Australia, priority-driven Collaborative Cancer Research Scheme (1147276). The content is solely the responsibility of the individual authors and does not reflect the views of Cancer Australia.

Conflicts of Interest: The authors declare no conflict of interest.

\section{References}

1. Bray, F.; Ferlay, J.; Soerjomataram, I.; Siegel, R.L.; Torre, L.A.; Jemal, A. Global cancer statistics 2018: GLOBOCAN estimates of incidence and mortality worldwide for 36 cancers in 185 countries. CA Cancer J. Clin. 2018, 68, 394-424. [CrossRef]

2. Prat, J. Ovarian carcinomas: Five distinct diseases with different origins, genetic alterations, and clinicopathological features. Virchows Arch. 2012, 460, 237-249. [CrossRef]

3. Torre, L.A.; Trabert, B.; DeSantis, C.E.; Miller, K.D.; Samimi, G.; Runowicz, C.D.; Gaudet, M.M.; Jemal, A.; Siegel, R.L. Ovarian cancer statistics, 2018. CA Cancer J. Clin. 2018, 68, 284-296. [CrossRef]

4. Buys, S.S.; Partridge, E.; Black, A.; Johnson, C.C.; Lamerato, L.; Isaacs, C.; Reding, D.J.; Greenlee, R.T.; Yokochi, L.A.; Kessel, B.; et al. Effect of screening on ovarian cancer mortality: The Prostate, Lung, Colorectal and Ovarian (PLCO) Cancer Screening Randomized Controlled Trial. JAMA 2011, 305, 2295-2303. [CrossRef]

5. Jacobs, I.J.; Menon, U.; Ryan, A.; Gentry-Maharaj, A.; Burnell, M.; Kalsi, J.K.; Amso, N.N.; Apostolidou, S.; Benjamin, E.; Cruickshank, D.; et al. Ovarian cancer screening and mortality in the UK Collaborative Trial of Ovarian Cancer Screening (UKCTOCS): A randomised controlled trial. Lancet 2016, 387, 945-956. [CrossRef]

6. Jayson, G.C.; Kohn, E.C.; Kitchener, H.C.; Ledermann, J.A. Ovarian cancer. Lancet 2014, 384, 1376-1388. [CrossRef]

7. Thomas, D.M.; James, P.A.; Ballinger, M.L. Clinical implications of genomics for cancer risk genetics. Lancet Oncol. 2015, 16, e303-e308. [CrossRef]

8. Jervis, S.; Song, H.; Lee, A.; Dicks, E.; Tyrer, J.; Harrington, P.; Easton, D.F.; Jacobs, I.J.; Pharoah, P.P.; Antoniou, A.C. Ovarian cancer familial relative risks by tumour subtypes and by known ovarian cancer genetic susceptibility variants. J. Med. Genet. 2014, 51, 108-113. [CrossRef]

9. Kuchenbaecker, K.B.; Hopper, J.L.; Barnes, D.R.; Phillips, K.A.; Mooij, T.M.; Roos-Blom, M.J.; Jervis, S.; van Leeuwen, F.E.; Milne, R.L.; Andrieu, N.; et al. Risks of Breast, Ovarian, and Contralateral Breast Cancer for BRCA1 and BRCA2 Mutation Carriers. JAMA 2017, 317, 2402-2416. [CrossRef]

10. Yang, X.; Song, H.; Leslie, G.; Engel, C.; Hahnen, E.; Auber, B.; Horváth, J.; Kast, K.; Niederacher, D.; Turnbull, C.; et al. Ovarian and Breast Cancer Risks Associated With Pathogenic Variants in RAD51C and RAD51D. J. Natl. Cancer Inst. 2020, 112. [CrossRef]

11. Ramus, S.J.; Song, H.; Dicks, E.; Tyrer, J.P.; Rosenthal, A.N.; Intermaggio, M.P.; Fraser, L.; Gentry-Maharaj, A.; Hayward, J.; Philpott, S.; et al. Germline Mutations in the BRIP1, BARD1, PALB2, and NBN Genes in Women With Ovarian Cancer. J. Natl. Cancer Inst. 2015, 107. [CrossRef]

12. Yang, X.; Leslie, G.; Doroszuk, A.; Schneider, S.; Allen, J.; Decker, B.; Dunning, A.M.; Redman, J.; Scarth, J.; Plaskocinska, I.; et al. Cancer Risks Associated with Germline PALB2 Pathogenic Variants: An International Study of 524 Families. J. Clin. Oncol. 2020, 38, 674-685. [CrossRef]

13. Møller, P.; Seppälä, T.T.; Bernstein, I.; Holinski-Feder, E.; Sala, P.; Gareth Evans, D.; Lindblom, A.; Macrae, F.; Blanco, I.; Sijmons, R.H.; et al. Cancer risk and survival in path_MMR carriers by gene and gender up to 75 years of age: A report from the Prospective Lynch Syndrome Database. Gut 2018, 67, 1306-1316. [CrossRef] 
14. Kar, S.P.; Berchuck, A.; Gayther, S.A.; Goode, E.L.; Moysich, K.B.; Pearce, C.L.; Ramus, S.J.; Schildkraut, J.M.; Sellers, T.A.; Pharoah, P.D.P. Common Genetic Variation and Susceptibility to Ovarian Cancer: Current Insights and Future Directions. Cancer Epidemiol. Biomark. Prev. 2018, 27, 395. [CrossRef]

15. Phelan, C.M.; Kuchenbaecker, K.B.; Tyrer, J.P.; Kar, S.P.; Lawrenson, K.; Winham, S.J.; Dennis, J.; Pirie, A.; Riggan, M.J.; Chornokur, G.; et al. Identification of 12 new susceptibility loci for different histotypes of epithelial ovarian cancer. Nat. Genet. 2017, 49, 680-691. [CrossRef]

16. Ramus, S.J.; Harrington, P.A.; Pye, C.; DiCioccio, R.A.; Cox, M.J.; Garlinghouse-Jones, K.; Oakley-Girvan, I.; Jacobs, I.J.; Hardy, R.M.; Whittemore, A.S.; et al. Contribution of BRCA1 and BRCA2 mutations to inherited ovarian cancer. Hum. Mutat. 2007, 28, 1207-1215. [CrossRef]

17. Ramus, S.J.; Gayther, S.A. The Contribution of BRCA1 and BRCA2 to Ovarian Cancer. Mol. Oncol. 2009, 3, 138-150. [CrossRef]

18. Johannesdottir, G.; Gudmundsson, J.; Bergthorsson, J.T.; Arason, A.; Agnarsson, B.A.; Eiriksdottir, G.; Johannsson, O.T.; Borg, A.; Ingvarsson, S.; Easton, D.F.; et al. High Prevalence of the 999del5 Mutation in Icelandic Breast and Ovarian Cancer Patients. Cancer Res. 1996, 56, 3663-3665.

19. Gayther, S.A.; Harrington, P.; Russell, P.; Kharkevich, G.; Garkavtseva, R.F.; Ponder, B.A. Frequently occurring germ-line mutations of the BRCA1 gene in ovarian cancer families from Russia. Am. J. Hum. Genet. 1997, 60, 1239-1242.

20. Sokolenko, A.P.; Rozanov, M.E.; Mitiushkina, N.V.; Sherina, N.Y.; Iyevleva, A.G.; Chekmariova, E.V.; Buslov, K.G.; Shilov, E.S.; Togo, A.V.; Bit-Sava, E.M.; et al. Founder mutations in early-onset, familial and bilateral breast cancer patients from Russia. Fam. Cancer 2007, 6, 281-286. [CrossRef] [PubMed]

21. Menkiszak, J.; Gronwald, J.; Górski, B.; Jakubowska, A.; Huzarski, T.; Byrski, T.; Foszczyńska-Kłoda, M.; Haus, O.; Janiszewska, H.; Perkowska, M.; et al. Hereditary ovarian cancer in Poland. Int. J. Cancer 2003, 106, 942-945. [CrossRef]

22. Heimdal, K.; Maehle, L.; Apold, J.; Pedersen, J.C.; Møller, P. The Norwegian founder mutations in BRCA1: High penetrance confirmed in an incident cancer series and differences observed in the risk of ovarian cancer. Eur. J. Cancer 2003, 39, 2205-2213. [CrossRef]

23. Sarantaus, L.; Huusko, P.; Eerola, H.; Launonen, V.; Vehmanen, P.; Rapakko, K.; Gillanders, E.; Syrjäkoski, K.; Kainu, T.; Vahteristo, P.; et al. Multiple founder effects and geographical clustering of BRCA1 and BRCA2 families in Finland. Eur. J. Hum. Genet. 2000, 8, 757-763. [CrossRef]

24. Sekine, M.; Nagata, H.; Tsuji, S.; Hirai, Y.; Fujimoto, S.; Hatae, M.; Kobayashi, I.; Fujii, T.; Nagata, I.; Ushijima, K.; et al. Mutational analysis of BRCA1 and BRCA2 and clinicopathologic analysis of ovarian cancer in 82 ovarian cancer families: Two common founder mutations of BRCA1 in Japanese population. Clin. Cancer Res. 2001, 7, 3144-3150.

25. Khoo, U.S.; Chan, K.Y.; Cheung, A.N.; Xue, W.C.; Shen, D.H.; Fung, K.Y.; Ngan, H.Y.; Choy, K.W.; Pang, C.P.; Poon, C.S.; et al. Recurrent BRCA1 and BRCA2 germline mutations in ovarian cancer: A founder mutation of BRCA1 identified in the Chinese population. Hum. Mutat. 2002, 19, 307-308. [CrossRef]

26. Kurian, A.W.; Hughes, E.; Handorf, E.A.; Gutin, A.; Allen, B.; Hartman, A.-R.; Hall, M.J. Breast and Ovarian Cancer Penetrance Estimates Derived from Germline Multiple-Gene Sequencing Results in Women. JCO Precis. Oncol. 2017, 1, 1-12. [CrossRef]

27. Lilyquist, J.; LaDuca, H.; Polley, E.; Davis, B.T.; Shimelis, H.; Hu, C.; Hart, S.N.; Dolinsky, J.S.; Couch, F.J.; Goldgar, D.E. Frequency of mutations in a large series of clinically ascertained ovarian cancer cases tested on multi-gene panels compared to reference controls. Gynecol. Oncol. 2017, 147, 375-380. [CrossRef]

28. Castéra, L.; Harter, V.; Muller, E.; Krieger, S.; Goardon, N.; Ricou, A.; Rousselin, A.; Paimparay, G.; Legros, A.; Bruet, O.; et al. Landscape of pathogenic variations in a panel of 34 genes and cancer risk estimation from 5131 HBOC families. Genet. Med. 2018, 20, 1677-1686. [CrossRef]

29. Suszynska, M.; Klonowska, K.; Jasinska, A.J.; Kozlowski, P. Large-scale meta-analysis of mutations identified in panels of breast/ovarian cancer-related genes-Providing evidence of cancer predisposition genes. Gynecol. Oncol. 2019, 153, 452-462. [CrossRef]

30. Iqbal, J.; Ragone, A.; Lubinski, J.; Lynch, H.T.; Moller, P.; Ghadirian, P.; Foulkes, W.D.; Armel, S.; Eisen, A.; Neuhausen, S.L.; et al. The incidence of pancreatic cancer in BRCA1 and BRCA2 mutation carriers. Br. J. Cancer 2012, 107, 2005-2009. [CrossRef] 
31. Pritchard, C.C.; Mateo, J.; Walsh, M.F.; De Sarkar, N.; Abida, W.; Beltran, H.; Garofalo, A.; Gulati, R.; Carreira, S.; Eeles, R.; et al. Inherited DNA-Repair Gene Mutations in Men with Metastatic Prostate Cancer. N. Engl. J. Med. 2016, 375, 443-453. [CrossRef]

32. Alsop, K.; Fereday, S.; Meldrum, C.; deFazio, A.; Emmanuel, C.; George, J.; Dobrovic, A.; Birrer, M.J.; Webb, P.M.; Stewart, C.; et al. BRCA mutation frequency and patterns of treatment response in BRCA mutation-positive women with ovarian cancer: A report from the Australian Ovarian Cancer Study Group. J. Clin. Oncol. 2012, 30, 2654-2663. [CrossRef]

33. Song, H.; Cicek, M.S.; Dicks, E.; Harrington, P.; Ramus, S.J.; Cunningham, J.M.; Fridley, B.L.; Tyrer, J.P.; Alsop, J.; Jimenez-Linan, M.; et al. The contribution of deleterious germline mutations in BRCA1, BRCA2 and the mismatch repair genes to ovarian cancer in the population. Hum. Mol. Genet. 2014, 23, 4703-4709. [CrossRef]

34. Walsh, T.; Casadei, S.; Lee, M.K.; Pennil, C.C.; Nord, A.S.; Thornton, A.M.; Roeb, W.; Agnew, K.J.; Stray, S.M.; Wickramanayake, A.; et al. Mutations in 12 genes for inherited ovarian, fallopian tube, and peritoneal carcinoma identified by massively parallel sequencing. Proc. Natl. Acad. Sci. USA 2011, 108, 18032-18037. [CrossRef]

35. Norquist, B.M.; Harrell, M.I.; Brady, M.F.; Walsh, T.; Lee, M.K.; Gulsuner, S.; Bernards, S.S.; Casadei, S.; Yi, Q.; Burger, R.A.; et al. Inherited Mutations in Women with Ovarian Carcinoma. JAMA Oncol. 2016, 2, 482-490. [CrossRef]

36. Bolton, K.L.; Chenevix-Trench, G.; Goh, C.; Sadetzki, S.; Ramus, S.J.; Karlan, B.Y.; Lambrechts, D.; Despierre, E.; Barrowdale, D.; McGuffog, L.; et al. Association between BRCA1 and BRCA2 Mutations and Survival in Women with Invasive Epithelial Ovarian Cancer. JAMA 2012, 307, 382-390. [CrossRef]

37. Kotsopoulos, J.; Rosen, B.; Fan, I.; Moody, J.; McLaughlin, J.R.; Risch, H.; May, T.; Sun, P.; Narod, S.A. Ten-year survival after epithelial ovarian cancer is not associated with BRCA mutation status. Gynecol. Oncol. 2016, 140, 42-47. [CrossRef]

38. McLaughlin, J.R.; Rosen, B.; Moody, J.; Pal, T.; Fan, I.; Shaw, P.A.; Risch, H.A.; Sellers, T.A.; Sun, P.; Narod, S.A. Long-Term Ovarian Cancer Survival Associated with Mutation in BRCA1 or BRCA2. J. Natl. Cancer Inst. 2013, 105, 141-148. [CrossRef]

39. National Comprehensive Cancer Network. Genetics Screening (Version 2.2019). Available online: https: //www.nccn.org/professionals/physician_gls/pdf/genetics_screening.pdf (accessed on 11 June 2020).

40. Cancer Genome Atlas Research Network. Integrated genomic analyses of ovarian carcinoma. Nature 2011, 474, 609-615. [CrossRef]

41. Kaufman, B.; Shapira-Frommer, R.; Schmutzler, R.K.; Audeh, M.W.; Friedlander, M.; Balmaña, J.; Mitchell, G.; Fried, G.; Stemmer, S.M.; Hubert, A.; et al. Olaparib monotherapy in patients with advanced cancer and a germline BRCA1/2 mutation. J. Clin. Oncol. 2015, 33, 244-250. [CrossRef]

42. Swisher, E.M.; Lin, K.K.; Oza, A.M.; Scott, C.L.; Giordano, H.; Sun, J.; Konecny, G.E.; Coleman, R.L.; Tinker, A.V.; O'Malley, D.M.; et al. Rucaparib in relapsed, platinum-sensitive high-grade ovarian carcinoma (ARIEL2 Part 1): An international, multicentre, open-label, phase 2 trial. Lancet Oncol. 2017, 18, 75-87. [CrossRef]

43. Oza, A.M.; Tinker, A.V.; Oaknin, A.; Shapira-Frommer, R.; McNeish, I.A.; Swisher, E.M.; Ray-Coquard, I.; Bell-McGuinn, K.; Coleman, R.L.; O'Malley, D.M.; et al. Antitumor activity and safety of the PARP inhibitor rucaparib in patients with high-grade ovarian carcinoma and a germline or somatic BRCA1 or BRCA2 mutation: Integrated analysis of data from Study 10 and ARIEL2. Gynecol. Oncol. 2017, 147, 267-275. [CrossRef]

44. Gelmon, K.A.; Tischkowitz, M.; Mackay, H.; Swenerton, K.; Robidoux, A.; Tonkin, K.; Hirte, H.; Huntsman, D.; Clemons, M.; Gilks, B.; et al. Olaparib in patients with recurrent high-grade serous or poorly differentiated ovarian carcinoma or triple-negative breast cancer: A phase 2, multicentre, open-label, non-randomised study. Lancet Oncol. 2011, 12, 852-861. [CrossRef]

45. Suszynska, M.; Ratajska, M.; Kozlowski, P. BRIP1, RAD51C, and RAD51D mutations are associated with high susceptibility to ovarian cancer: Mutation prevalence and precise risk estimates based on a pooled analysis of $\sim 30,000$ cases. J. Ovarian Res. 2020, 13, 50. [CrossRef]

46. Meindl, A.; Hellebrand, H.; Wiek, C.; Erven, V.; Wappenschmidt, B.; Niederacher, D.; Freund, M.; Lichtner, P.; Hartmann, L.; Schaal, H.; et al. Germline mutations in breast and ovarian cancer pedigrees establish RAD51C as a human cancer susceptibility gene. Nat. Genet. 2010, 42, 410-414. [CrossRef] 
47. Loveday, C.; Turnbull, C.; Ramsay, E.; Hughes, D.; Ruark, E.; Frankum, J.R.; Bowden, G.; Kalmyrzaev, B.; Warren-Perry, M.; Snape, K.; et al. Germline mutations in RAD51D confer susceptibility to ovarian cancer. Nat. Genet. 2011, 43, 879-882. [CrossRef]

48. Song, H.; Dicks, E.; Ramus, S.J.; Tyrer, J.P.; Intermaggio, M.P.; Hayward, J.; Edlund, C.K.; Conti, D.; Harrington, P.; Fraser, L.; et al. Contribution of Germline Mutations in the RAD51B, RAD51C, and RAD51D Genes to Ovarian Cancer in the Population. J. Clin. Oncol. 2015, 33, 2901-2907. [CrossRef]

49. Manchanda, R.; Legood, R.; Antoniou, A.; Pearce, L.; Menon, U. Commentary on changing the risk threshold for surgical prevention of ovarian cancer. BJOG An. Int. J. Obstet. Gynaecol. 2018, 125, 541-544. [CrossRef]

50. Manchanda, R.; Legood, R.; Antoniou, A.C.; Gordeev, V.S.; Menon, U. Specifying the ovarian cancer risk threshold of 'premenopausal risk-reducing salpingo-oophorectomy' for ovarian cancer prevention: A cost-effectiveness analysis. J. Med. Genet. 2016, 53, 591-599. [CrossRef]

51. Manchanda, R.; Legood, R.; Pearce, L.; Menon, U. Defining the risk threshold for risk reducing salpingo-oophorectomy for ovarian cancer prevention in low risk postmenopausal women. Gynecol. Oncol. 2015, 139, 487-494. [CrossRef]

52. Antoniou, A.C.; Casadei, S.; Heikkinen, T.; Barrowdale, D.; Pylkas, K.; Roberts, J.; Lee, A.; Subramanian, D.; De Leeneer, K.; Fostira, F.; et al. Breast-cancer risk in families with mutations in PALB2. N. Engl. J. Med. 2014, 371, 497-506. [CrossRef]

53. Ducy, M.; Sesma-Sanz, L.; Guitton-Sert, L.; Lashgari, A.; Gao, Y.; Brahiti, N.; Rodrigue, A.; Margaillan, G.; Caron, M.C.; Côté, J.; et al. The Tumor Suppressor PALB2: Inside out. Trends Biochem. Sci. 2019, 44, 226-240. [CrossRef]

54. Song, H.; Ramus, S.; Dicks, E.; Tyrer, J.; Intermaggio, M.; Chenevix-Trench, G.; Bowtell, D.; Traficante, N.; Brenton, J.; Goranova, T.; et al. Population based targeted sequencing of 54 candidate genes identifies PALB2 as a susceptibility gene for high grade serous ovarian cancer. J Med Genet. 2020. [CrossRef]

55. Osorio, A.; Endt, D.; Fernández, F.; Eirich, K.; de la Hoya, M.; Schmutzler, R.; Caldés, T.; Meindl, A.; Schindler, D.; Benitez, J. Predominance of pathogenic missense variants in the RAD51C gene occurring in breast and ovarian cancer families. Hum. Mol. Genet. 2012, 21, 2889-2898. [CrossRef]

56. Ketabi, Z.; Bartuma, K.; Bernstein, I.; Malander, S.; Grönberg, H.; Björck, E.; Holck, S.; Nilbert, M. Ovarian cancer linked to Lynch syndrome typically presents as early-onset, non-serous epithelial tumors. Gynecol. Oncol. 2011, 121, 462-465. [CrossRef]

57. Lu, H.M.; Li, S.; Black, M.H.; Lee, S.; Hoiness, R.; Wu, S.; Mu, W.; Huether, R.; Chen, J.; Sridhar, S.; et al. Association of Breast and Ovarian Cancers with Predisposition Genes Identified by Large-Scale Sequencing. JAMA Oncol. 2019, 5, 51-57. [CrossRef]

58. Spurdle, A.B.; Healey, S.; Devereau, A.; Hogervorst, F.B.; Monteiro, A.N.; Nathanson, K.L.; Radice, P.; Stoppa-Lyonnet, D.; Tavtigian, S.; Wappenschmidt, B.; et al. ENIGMA-Evidence-based network for the interpretation of germline mutant alleles: An international initiative to evaluate risk and clinical significance associated with sequence variation in BRCA1 and BRCA2 genes. Hum. Mutat 2012, 33, 2-7. [CrossRef]

59. Li, H.; LaDuca, H.; Pesaran, T.; Chao, E.C.; Dolinsky, J.S.; Parsons, M.; Spurdle, A.B.; Polley, E.C.; Shimelis, H.; Hart, S.N.; et al. Classification of variants of uncertain significance in BRCA1 and BRCA2 using personal and family history of cancer from individuals in a large hereditary cancer multigene panel testing cohort. Genet. Med. 2020, 22, 701-708. [CrossRef]

60. Thompson, B.A.; Spurdle, A.B.; Plazzer, J.P.; Greenblatt, M.S.; Akagi, K.; Al-Mulla, F.; Bapat, B.; Bernstein, I.; Capellá, G.; den Dunnen, J.T.; et al. Application of a 5-tiered scheme for standardized classification of 2,360 unique mismatch repair gene variants in the InSiGHT locus-specific database. Nat. Genet. 2014, 46, 107-115. [CrossRef]

61. Ng, P.C.; Henikoff, S. SIFT: Predicting amino acid changes that affect protein function. Nucleic Acids Res. 2003, 31, 3812-3814. [CrossRef]

62. Adzhubei, I.; Jordan, D.M.; Sunyaev, S.R. Predicting functional effect of human missense mutations using PolyPhen-2. Curr. Protoc. Hum. Genet. 2013, 76, 7-20. [CrossRef]

63. Choi, Y.; Chan, A.P. PROVEAN web server: A tool to predict the functional effect of amino acid substitutions and indels. Bioinformatics 2015, 31, 2745-2747. [CrossRef]

64. Tyrer, J.P.; Guo, Q.; Easton, D.F.; Pharoah, P.D. The admixture maximum likelihood test to test for association between rare variants and disease phenotypes. BMC Bioinform. 2013, 14, 177. [CrossRef] 
65. Bouwman, P.; van der Gulden, H.; van der Heijden, I.; Drost, R.; Klijn, C.N.; Prasetyanti, P.; Pieterse, M.; Wientjens, E.; Seibler, J.; Hogervorst, F.B.; et al. A high-throughput functional complementation assay for classification of BRCA1 missense variants. Cancer Discov. 2013, 3, 1142-1155. [CrossRef]

66. Dicks, E.; Song, H.; Ramus, S.J.; Oudenhove, E.V.; Tyrer, J.P.; Intermaggio, M.P.; Kar, S.; Harrington, P.; Bowtell, D.D.; Group, A.S.; et al. Germline whole exome sequencing and large-scale replication identifies FANCM as a likely high grade serous ovarian cancer susceptibility gene. Oncotarget 2017, 8, 50930-50940. [CrossRef]

67. Thompson, D.; Duedal, S.; Kirner, J.; McGuffog, L.; Last, J.; Reiman, A.; Byrd, P.; Taylor, M.; Easton, D.F. Cancer Risks and Mortality in Heterozygous ATM Mutation Carriers. JNCI J. Natl. Cancer Inst. 2005, 97, 813-822. [CrossRef]

68. Roberts, N.J.; Jiao, Y.; Yu, J.; Kopelovich, L.; Petersen, G.M.; Bondy, M.L.; Gallinger, S.; Schwartz, A.G.; Syngal, S.; Cote, M.L.; et al. ATM mutations in patients with hereditary pancreatic cancer. Cancer Discov. 2012, 2, 41-46. [CrossRef]

69. Goldgar, D.E.; Healey, S.; Dowty, J.G.; Da Silva, L.; Chen, X.; Spurdle, A.B.; Terry, M.B.; Daly, M.J.; Buys, S.M.; Southey, M.C.; et al. Rare variants in the ATM gene and risk of breast cancer. Breast Cancer Res. 2011, 13, R73. [CrossRef]

70. Weber-Lassalle, N.; Borde, J.; Weber-Lassalle, K.; Horváth, J.; Niederacher, D.; Arnold, N.; Kaulfuß, S.; Ernst, C.; Paul, V.G.; Honisch, E.; et al. Germline loss-of-function variants in the BARD1 gene are associated with early-onset familial breast cancer but not ovarian cancer. Breast Cancer Res. 2019, 21, 55. [CrossRef]

71. Couch, F.J.; Shimelis, H.; Hu, C.; Hart, S.N.; Polley, E.C.; Na, J.; Hallberg, E.; Moore, R.; Thomas, A.; Lilyquist, J.; et al. Associations between Cancer Predisposition Testing Panel Genes and Breast Cancer. JAMA Oncol. 2017, 3, 1190-1196. [CrossRef]

72. Weischer, M.; Nordestgaard, B.G.; Pharoah, P.; Bolla, M.K.; Nevanlinna, H.; Van't Veer, L.J.; Garcia-Closas, M.; Hopper, J.L.; Hall, P.; Andrulis, I.L.; et al. CHEK2*1100delC heterozygosity in women with breast cancer associated with early death, breast cancer-specific death, and increased risk of a second breast cancer. J. Clin. Oncol. Off. J. Am. Soc. Clin. Oncol. 2012, 30, 4308-4316. [CrossRef]

73. Gylling, A.; Ridanpää, M.; Vierimaa, O.; Aittomäki, K.; Avela, K.; Kääriäinen, H.; Laivuori, H.; Pöyhönen, M.; Sallinen, S.-L.; Wallgren-Pettersson, C.; et al. Large genomic rearrangements and germline epimutations in Lynch syndrome. Int. J. Cancer 2009, 124, 2333-2340. [CrossRef]

74. Easton, D.F.; Pharoah, P.D.; Antoniou, A.C.; Tischkowitz, M.; Tavtigian, S.V.; Nathanson, K.L.; Devilee, P.; Meindl, A.; Couch, F.J.; Southey, M.; et al. Gene-panel sequencing and the prediction of breast-cancer risk. N. Engl. J. Med. 2015, 372, 2243-2257. [CrossRef] [PubMed]

75. Prapa, M.; Solomons, J.; Tischkowitz, M. The use of panel testing in familial breast and ovarian cancer. Clin. Med. 2017, 17, 568-572. [CrossRef]

76. Menon, U.; Karpinskyj, C.; Gentry-Maharaj, A. Ovarian Cancer Prevention and Screening. Obstet Gynecol. 2018, 131, 909-927. [CrossRef]

77. Domchek, S.M.; Robson, M.E. Update on Genetic Testing in Gynecologic Cancer. J. Clin. Oncol. 2019, 37, 2501-2509. [CrossRef]

78. Manchanda, R.; Menon, U. Setting the Threshold for Surgical Prevention in Women at Increased Risk of Ovarian Cancer. Int. J. Gynecol. Cancer 2018, 28, 34-42. [CrossRef]

79. Rosenthal, A.N.; Fraser, L.S.M.; Philpott, S.; Manchanda, R.; Burnell, M.; Badman, P.; Hadwin, R.; Rizzuto, I.; Benjamin, E.; Singh, N.; et al. Evidence of Stage Shift in Women Diagnosed with Ovarian Cancer during Phase II of the United Kingdom Familial Ovarian Cancer Screening Study. J. Clin. Oncol. 2017, 35, 1411-1420. [CrossRef] [PubMed]

80. Clayton, E.W. Ethical, legal, and social implications of genomic medicine. N. Engl. J. Med. 2003, 349, 562-569. [CrossRef]

81. Mersch, J.; Brown, N.; Pirzadeh-Miller, S.; Mundt, E.; Cox, H.C.; Brown, K.; Aston, M.; Esterling, L.; Manley, S.; Ross, T. Prevalence of Variant Reclassification Following Hereditary Cancer Genetic Testing. JAMA 2018, 320, 1266-1274. [CrossRef]

82. LaDuca, H.; Stuenkel, A.J.; Dolinsky, J.S.; Keiles, S.; Tandy, S.; Pesaran, T.; Chen, E.; Gau, C.-L.; Palmaer, E.; Shoaepour, K.; et al. Utilization of multigene panels in hereditary cancer predisposition testing: Analysis of more than 2,000 patients. Genet. Med. Off. J. Am. Coll. Med Genet. 2014, 16, 830-837. [CrossRef] [PubMed] 
83. Tandy-Connor, S.; Guiltinan, J.; Krempely, K.; LaDuca, H.; Reineke, P.; Gutierrez, S.; Gray, P.; Tippin Davis, B. False-positive results released by direct-to-consumer genetic tests highlight the importance of clinical confirmation testing for appropriate patient care. Genet. Med. 2018, 20, 1515-1521. [CrossRef] [PubMed]

84. Tung, N.; Domchek, S.M.; Stadler, Z.; Nathanson, K.L.; Couch, F.; Garber, J.E.; Offit, K.; Robson, M.E. Counselling framework for moderate-penetrance cancer-susceptibility mutations. Nat. Rev. Clin. Oncol. 2016, 13, 581-588. [CrossRef] [PubMed]

85. Giuliano, A.E.; Boolbol, S.; Degnim, A.; Kuerer, H.; Leitch, A.M.; Morrow, M. Society of Surgical Oncology: Position statement on prophylactic mastectomy. Approved by the Society of Surgical Oncology Executive Council, March 2007. Ann. Surg Oncol. 2007, 14, 2425-2427. [CrossRef] [PubMed]

86. Gabai-Kapara, E.; Lahad, A.; Kaufman, B.; Friedman, E.; Segev, S.; Renbaum, P.; Beeri, R.; Gal, M.; Grinshpun-Cohen, J.; Djemal, K.; et al. Population-based screening for breast and ovarian cancer risk due to BRCA1 and BRCA2. Proc. Natl. Acad. Sci. USA 2014, 111, 14205-14210. [CrossRef] [PubMed]

87. Manchanda, R.; Loggenberg, K.; Sanderson, S.; Burnell, M.; Wardle, J.; Gessler, S.; Side, L.; Balogun, N.; Desai, R.; Kumar, A.; et al. Population Testing for Cancer Predisposing BRCA1/BRCA2 Mutations in the Ashkenazi-Jewish Community: A Randomized Controlled Trial. JNCI J. Natl. Cancer Inst. 2014, 107. [CrossRef] [PubMed]

88. Chen, K.; Ma, H.; Li, L.; Zang, R.; Wang, C.; Song, F.; Shi, T.; Yu, D.; Yang, M.; Xue, W.; et al. Genome-wide association study identifies new susceptibility loci for epithelial ovarian cancer in Han Chinese women. Nat. Commun. 2014, 5, 4682. [CrossRef]

89. Coleman, R.L.; Fleming, G.F.; Brady, M.F.; Swisher, E.M.; Steffensen, K.D.; Friedlander, M.; Okamoto, A.; Moore, K.N.; Efrat Ben-Baruch, N.; Werner, T.L.; et al. Veliparib with First-Line Chemotherapy and as Maintenance Therapy in Ovarian Cancer. N. Engl. J. Med. 2019, 381, 2403-2415. [CrossRef]

90. Ray-Coquard, I.; Pautier, P.; Pignata, S.; Pérol, D.; González-Martín, A.; Berger, R.; Fujiwara, K.; Vergote, I.; Colombo, N.; Mäenpää, J.; et al. Olaparib plus Bevacizumab as First-Line Maintenance in Ovarian Cancer. N. Engl. J. Med. 2019, 381, 2416-2428. [CrossRef]

91. González-Martín, A.; Pothuri, B.; Vergote, I.; DePont Christensen, R.; Graybill, W.; Mirza, M.R.; McCormick, C.; Lorusso, D.; Hoskins, P.; Freyer, G.; et al. Niraparib in Patients with Newly Diagnosed Advanced Ovarian Cancer. N. Engl. J. Med. 2019, 381, 2391-2402. [CrossRef]

92. Longo, D.L. Personalized Medicine for Primary Treatment of Serous Ovarian Cancer. N. Engl. J. Med. 2019, 381, 2471-2474. [CrossRef] [PubMed]

93. Lheureux, S.; Gourley, C.; Vergote, I.; Oza, A.M. Epithelial ovarian cancer. Lancet 2019, 393, 1240-1253. [CrossRef]

Publisher's Note: MDPI stays neutral with regard to jurisdictional claims in published maps and institutional affiliations.

(C) 2020 by the authors. Licensee MDPI, Basel, Switzerland. This article is an open access article distributed under the terms and conditions of the Creative Commons Attribution (CC BY) license (http://creativecommons.org/licenses/by/4.0/). 